\title{
Effect of Arbuscular mycorrhizal Fungal Colonization on Nutrient Uptake in Rice Aerobic Conditions
}

\author{
Ekta Narwal ${ }^{1}$, K. Annapurna ${ }^{1 *}$, Jairam Choudhary ${ }^{2}$ and Seema Sangwan ${ }^{1}$ \\ ${ }^{1}$ ICAR-Indian Agricultural Research Institute, New Delhi-110012, India \\ ${ }^{2}$ ICAR-Indian Institute of Farming Systems Research, Modipuram-250110, India \\ *Corresponding author
}

\begin{tabular}{l} 
K e y w o r d s \\
$\begin{array}{l}\text { Arbuscularmycorrhiza } \\
\text { (AM), Plant nutrient } \\
\text { uptake, Chlorophyll, } \\
\text { Nitrate redutase (NR) }\end{array}$ \\
\hline Article Info \\
$\begin{array}{l}\text { Accepted: } \\
10 \text { March } 2018 \\
\text { Available Online: } \\
10 \text { April } 2018\end{array}$ \\
\hline
\end{tabular}

Ten upland rice varieties were grown under aerobic conditions in the fields of IARI, New Delhi and were studied for mycorrhizal status and their growth performance. Rice varieties, Satyabhama and Pyari were shown to have highest response towards mycorrhizal colonization. Percentage of arbuscular mycorrhizal fungi (AMF) root colonization varied between $26.60 \%$ and $55.40 \%$. Greater plant nitrogen uptake $\left(110.70 \mathrm{kgha}^{-1}\right)$ and enhanced biomass $(54.9 \%)$ were observed with increased AMF colonization. Total $\mathrm{Zn}$ and Fe uptake by plants were higher in AMF treated rice by $32.4 \%$ and $22.4 \%$ compared to untreated control plants, respectively. $\mathrm{Zn}$ and Fe uptake was significantly increased by colonization with AMF. Increased biomass of rice plants can be attributed to improved P uptake (19.53 $\mathrm{kgha}^{-1}-12.22 \mathrm{kgha}^{-1}$ ) due to AM colonization. Mycorrhizal colonization showed positive correlation with the total chlorophyll content and nitrate reductase activity in the AMF treated rice. The study concludes that rice plants grown under aerobic conditions respond strongly to mycorrhizal infection and AM colonization greatly affects the plant nutrient status.

\section{Introduction}

About $90 \%$ of rice is produced and consumed in Asia, where the demand for rice is on the increase due to increasing population (Bouman, 2001). Rice is a semiaquatic plant and grows well under lowland flooded anaerobic conditions. Irrigated rice is the profligate user of fresh water as it utilizes 24$30 \%$ of the world's accessible freshwater resources (Singh, 2013). Toung and Bouman (2003) reported that by the year 2025 , about 17 million ha (mha) of irrigated rice area in Asia may experience 'physical water scarcity' and
22 mha may face 'economic water scarcity'. Rainfall patterns in many areas are becoming more unreliable, with extremes of drought and flooding occurring regularly. Thus, there is a need to economize water use in irrigated rice production. The transplanted puddled rice production system is labour, water and energy-intensive which proved less profitable (Kumar and Ladha, 2011).

The aerobic rice system (ARS) is a new production system in which rice is grown under non-puddled, non-flooded and nonsaturated soil conditions as other upland crops 
(Prasad, 2011; Bouman, 2001; Tuong and Bouman, 2003). Thus, in ARS, soils are kept aerobic almost throughout the rice-growing season. The driving force behind ARS is water economy and Castaneda et al., (2003) reported a saving of $73 \%$ in land preparation and $56 \%$ during crop growth. Yields obtained with ARS varieties vary from 4.5 to $6.5 \mathrm{t} / \mathrm{ha}$, which is 20-30\% lesser than that obtained with lowland varieties grown under flooded conditions (Farooq et al., 2009). The yields of aerobic rice gradually decline over time as compared to a continuously flooded control (Peng et al., 2006).

Nishizawa et al., (1971) introduced the term "soil sickness" for the combined effect of allelopathy (Nishio and Kusano, 1975), nutrient depletion (Lin et al., 2002), built-up of soil-borne diseases and pests (Ventura et al., 1981), and soil structure degradation. Thus, yield decline in aerobic rice monoculture over time could be due to both abiotic and biotic stresses.

Currently, there is a growing awareness of the importance of plant microbe interactions in plant performance, particularly for reducing nutrient inputs and minimize the environmental impacts of mineral fertilizers while maintaining plant growth and development (Jez et al., 2016). Soil microorganisms such as AM fungi symbiotically associated with plant roots and interacting with specific microbial communities are able to develop a range of activities to increase plant growth and crop productivity under stressed conditions (Barea et al., 2005; Azcón and Barea, 2010). Beneficial effect of microbial inoculants has been demonstrated in terms of greater biomass production, better nutrient uptake in plants and increase in grain $\mathrm{N}$ and $\mathrm{P}$ in rice grown under SRI practices (Prasanna et al., 2015) and increased micronutrient uptake (Adak et al., 2016).
Rice plants readily form mycorrhizal associations under upland conditions but, under submerged conditions, infection is rare due to the anoxic environment (Ilag et al., 1987). However, few reports have reported AMF colonization under flooded conditions (Secilia and Bagyaraj, 1994; Solaiman and Hirata, 1996). Thus, to obtain benefits from the AM symbiosis, rice should be grown under non-flooded conditions, creating aerobic conditions in the soil that stimulate colonization of rice roots by AM fungi (Vallino et al., 2009; Ruíz-Sánchez et al., 2010).

Rice can also be grown in a system of alternate irrigation to reduce water input needed for production; this system creates aerobic conditions in the soil that stimulates colonization of rice roots by AM fungi. However, a general view of AM fungal colonization of rice roots under aerobic conditions is not well documented, with a few studies demonstrating the deployment of AM fungi for the benefit of rice plants, under adverse environmental conditions (Vallino et al., 2009). From this perspective, the aim of this study was undertaken with hypotheses (i) AMF colonization in rice plants under aerobic conditions will enhance nutrient mobilization, (ii) AMF symbiosis has significant impact on the yield of rice and (iii) rice varieties differ significantly to the AMF colonization. The overall aim was to determine how this symbiosis influences plant growth, nutrient uptake and photosynthetic performance of rice plants under aerobic conditions.

\section{Materials and Methods}

\section{Experimental site and field management}

Ten upland rice varieties were grown under aerobic conditions in the fields of IARI, New Delhi. Details of the rice varieties used in the experiment are presented in Table 1. The 
experiment was conducted at the experimental farm of the ICAR-Indian Agricultural Research Institute (IARI), New Delhi, India $\left(28^{\circ} 40^{\prime} \mathrm{N}, 77^{\circ} 12^{\prime} \mathrm{E}, 228.6 \mathrm{~m}\right.$ above the mean sea-level), during the kharif season, 2015. The mean temperature was around $35^{\circ} \mathrm{C}$.Of the total recorded rainfall, 18.03, 256.18, 167.29, 19.2 and $2.22 \mathrm{~mm}$ were received during the month of June, July, August, September and October, respectively, in year 2015.

The experiment was laid out completely randomized design in three replicates with plot sizes $2 \mathrm{~m} \times 10 \mathrm{~m}$. Plots were separated by bunding. The plots were ploughed twice by disc harrow followed by one ploughing by cultivator, but no puddling was done. Aerobic plots were flash irrigated to keep the soil saturated with water. For sowing seeds, 20 rows were made in each plot, $25 \mathrm{~cm}$ apart and seeds of all the varieties were drilled at a depth of $2 \mathrm{~cm}$. A pre-sowing irrigation was given in the experimental field before sowing of the seed. Recommended doses of $\mathrm{N}$ (150kg/ha), $\mathrm{P}_{2} \mathrm{O}_{5}(60 \mathrm{~kg} / \mathrm{ha})$ and $\mathrm{K}_{2} \mathrm{O}(50 \mathrm{~kg} /$ ha) were applied to crops during the experiment in all the plots. Half dose of $\mathrm{N}$ and full doses of $\mathrm{P}$ and $\mathrm{K}$ were applied basal at the time of sowing and remaining $\mathrm{N}$ was applied in 2 equal splits, i.e. at tillering and panicleinitiation stages. The plots were kept weedfree by hand weeding. Irrigations to the crop were provided so as to keep soil near field capacity. Other crop-management practices were followed as per the recommendations. Mycorrhizal treatments included a nonmycorrhizal control and inoculation with the consortia of mycorrhizal fungus Glomus intraradices and Glomus mosseae. The mycorrhizal consortia was obtained from Division of Microbiology, ICAR-Indian Agricultural Research Institute, New Delhi, India. The inoculums consisted of colonized root segments and attached rhizosphere soil from Paspalum notatum were grown in a glasshouse.

\section{Chemical analysis of plant samples}

The plant samples from respective treatment plots were collected manually at the time of crop harvest. Ten rice plants were harvested from each plot and separated into grain and straw. Collected plant samples were dried in hot air oven at $60 \pm 2{ }^{\circ} \mathrm{C}$ for $6 \mathrm{~h}$. The ovendried samples of plants and air-dried samples of grains were ground to pass through 40mesh sieves in a macro-Wiley mill and analyzed for $\mathrm{N}, \mathrm{P}, \mathrm{K}, \mathrm{Zn}$ and $\mathrm{Fe}$ concentrations. Nitrogen, phosphorus and potassium concentrations were analyzed in grain and straw by adopting modified microKjeldahl method for nitrogen, vanadomolybdate yellow colour method for phosphorus and flame photometer method for potassium estimation as described by Jackson (1958). Fe concentration in rice grain or straw was analyzed by EDTA method. The nutrient uptake $\left(\mathrm{kg} \mathrm{ha}^{-1}\right)$ was computed by multiplying nitrogen, phosphorus, potassium and iron concentration with grain or straw yields $(\mathrm{kg}$ $\mathrm{ha}^{-1}$ ).

Chlorophyll content in aerobic rice flag leaves was determined at 30 DAS by the following procedure as depicted by Hiscox and Israelstam (1979). The chlorophyll $a$ and b, total chlorophyll content of leaf was computed using the formula suggested by Arnon (1949). One hundred milligram of leaf tissue was placed in a vial containing $7 \mathrm{ml}$ of DMSO and chlorophyll was extracted in to the fluid by incubating at $65^{\circ} \mathrm{C}$ overnight. The extract was then transferred to a graduated tube and made up to a total volume of $10 \mathrm{ml}$ with DMSO, assayed immediately or transferred to vials and stored between $0^{\circ} \mathrm{C}-40^{\circ} \mathrm{C}$ until required for analysis. Assay was done by transferring 3 $\mathrm{ml}$ of chlorophyll extract to a cuvette and the OD values at $645 \mathrm{~nm}$ and $663 \mathrm{~nm}$ were read in spectrophotometer against a DMSO blank. An in vitro method of nitrate reductase (NR) assay (Hageman and Reed, 1980) was used to 
estimate NR activity in the flag leaves. Crude protein content in rice grain was obtained by multiplying $\mathrm{N}$ concentration with a coefficient factor 5.85(Prasad et al., 2006). Mycorrhizal root colonization was determined by the method as described by Phillips and Haymann (1970). Briefly, $1 \mathrm{~cm}$ root samples were cleared in $10 \% \mathrm{KOH}$, acidified in $2 \% \mathrm{HCl}$ and stained with $0.05 \%$ trypan blue in lactophenol. Fifty root segments $(1 \mathrm{~cm}$ each) were randomly selected and mounted parallel to each other (ten each on glass slides). Every root segment was observed under microscope (200-400X) and rated according to the range of classes indicated by Trouvelot et al., (1986).

\section{Statistical analysis}

The data of all factors was analyzed statistically by standard analysis of variance (ANOVA) and differences were separated by least significant difference (LSD) using SAS version 9.3 (SAS Institute, Inc., Cary, NC, USA 1990). For statistical analysis of data, Microsoft-Excel software (Microsoft Corporation, USA) was used and significant differences were determined at $\operatorname{LSD}(\mathrm{P} \leq 0.05)$.

\section{Results and Discussion}

\section{Root colonization by AMF}

No root colonization by AMF was observed in uninoculated plants. There was a significant variation found in the root colonization among the rice varieties $(P \leq 0.05)$. Maximum root colonization with AMF was found at 30 DAS (Fig. 1). Root colonization was decreased by $65 \%$ and $120 \%$ at 45 and 60 DAS respectively, on an average. Rice variety, Satyabhama showed highest root colonization $(55.40 \%)$ followed by Pyari $(46.07 \%)$ at 30 DAS. Root colonization by AMF ranged from $26.60 \%$ to $55.40 \%$ at 30 DAS, which decreased from $16.07 \%-6.00 \%$ at 60 DAS.
Nutrient mobilization at flag leaf stage as influenced by AMF treatment

Nutrient concentration in the rice plants was significantly affected by AMF treatment (Table 3). Shoot N concentration was $67.3 \%$ higher in AMF treated rice plants as compared to untreated plants. Shoot $\mathrm{N}$ content ranged from $1.36 \%$ to $2.17 \%$, being highest found in Satyabhama $(2.17 \%)$ and lowest in DhalaHeera. Shoot $\mathrm{P}$ accumulation was significantly at par in untreated control plants.

In AMF treated rice plants, $\mathrm{P}$ content was found to be tremendously higher by $101 \%$. Shoot K content was also significantly affected by AMF treatment. There was a difference of about $70.3 \%$ in $\mathrm{K}$ accumulation in shoot between AMF treated and untreated control plants, being higher recorded in AMF treated rice. Maximum $\mathrm{K}$ content was observed in Satyabhama (2.51\%) followed by Pyari $(2.43 \%)$. AMF treatment significantly affected micronutrient accumulation. The $\mathrm{Zn}$ and $\mathrm{Fe}$ content in shoot were $28.4 \%$ and $15.04 \%$ higher in AMF treated plants, respectively. Shoot $\mathrm{Zn}$ concentration in AMF plants ranged from $36.46 \mathrm{mg} \mathrm{kg}^{-1}$ to $57.43 \mathrm{mg}$ $\mathrm{kg}^{-1}$. Highest Fe concentration was found in Satyabhama (159.79 $\mathrm{mg} \mathrm{kg}^{-1}$ ) and lowest in Anjali (31.99 $\left.\mathrm{mg} \mathrm{kg}^{-1}\right)$.

Chlorophyll content and Nitrate Reductase (NR) activity as influenced by AMF treatment

A $70.0 \%, 60.0 \%$ and $66.5 \%$ higher chlorophyll $a$, chlorophyll $b$ and total chlorophyll content respectively was observed in AMF treated rice plants. Total chlorophyll content in AMF rice ranged from $1.64 \mathrm{mg} \mathrm{g}^{-1}$ fresh weight to $3.26 \mathrm{mg} \mathrm{g}^{-1}$ fresh weight, highest being recorded for Satyabhama. The concentration of different chlorophyll pigments was found to be almost nonsignificant among control rice plants. 
Similarly, NR activity was significantly at par in control rice plants (Table 9). NR activity was found to be significantly higher in AMF treated rice plants. Highest NR among AMF treated rice was recorded for Satyabhama (41.12 $\mu$ moles of nitrite formed $\mathrm{h}^{-1} \mathrm{~g}^{-1}$ fresh weight) and CR Dhan204 exhibited lowest NR activity (25.25 $\mu$ moles of nitrite formed $\mathrm{h}^{-1} \mathrm{~g}^{-1}$ fresh weight). The interaction between rice varieties and AMF treatment was found to be significant.

Nitrogen $(\mathrm{N})$ concentration and uptake as influenced by AMF treatment

Nitrogen uptake differed significantly $(P \leq 0.05)$ between AMF treated and untreated rice (Table 4). Nitrogen concentration in both grain and straw was higher in AMF treated rice by $44.6 \%$ and $38.9 \%$, respectively as compared to control rice. AMF treated rice varieties Satyabhama and Pyari were recorded for highest $\mathrm{N}$ content in grain $(1.87 \%$ ) and straw $(0.62 \%)$ respectively. Satyabhama and Pyari were recorded for highest $\mathrm{N}$ uptake by grain $(74.80 \%)$ and straw $(44.64 \%)$, respectively. Total $\mathrm{N}$ uptake was higher in AM colonized rice by $41.8 \%$ compared to uninoculated plants. Among the non- AM treatments, Satyabhama showed maximum total $\mathrm{N}$ uptake $\left(110.70 \mathrm{~kg} \mathrm{ha}^{-1}\right)$ followed by CR Dhan $205\left(87.06 \mathrm{~kg} \mathrm{ha}^{-1}\right)$. There was a significant interaction between rice varieties and AMF treatment which is evident by the higher $\mathrm{N}$ concentration and uptake in AM-rice as compared to non-AM rice.

Phosphorus (P) concentration and uptake as influenced by AMF treatment

Rice variety Satyabhama was recorded for a significantly higher concentration of $\mathrm{P}$ in both grain $(0.37 \%)$ and straw $(0.24 \%)$ (Table 5). $\mathrm{P}$ content in grain was higher by $36.5 \%$ in AM treated rice. Similarly, $\mathrm{P}$ uptake in grain and straw was higher in AM rice by $37.8 \%$ and
$31.2 \%$ compared to non-AM rice. In control rice plants, $33.8 \%$ less total $\mathrm{P}$ uptake was observed. Satyabhama was recorded for highest $\mathrm{P}$ uptake in grain $\left(12.80 \mathrm{~kg} \mathrm{ha}^{-1}\right)$ and straw $\left(16.80 \mathrm{~kg} \mathrm{ha}^{-1}\right)$. The $\mathrm{P}$ concentration in grain and straw and its uptake was more or less at par in non- AM rice. The interactive effect of rice varieties with AMF shows that all the rice varieties accumulated higher $\mathrm{P}$ in grain and straw and their total when AM colonized.

\section{Potassium $(K)$ concentration and uptake as influenced by AMF treatment}

$\mathrm{K}$ concentration in grain was found to be nonsignificant among rice varieties whether AM colonized or not (Table 6), although K concentration in grain was higher in AM rice by $31.5 \%$ compared to non-AM rice. $\mathrm{K}$ concentration in straw was significantly at par among uninoculated plants. In AM colonized rice, Dhala Heera exhibited highest $\mathrm{K}$ accumulation $(1.62 \%)$ in straw. K uptake by grain in AM-rice ranged from $13.30 \mathrm{~kg} \mathrm{ha}^{-1}$ to $20.00 \mathrm{~kg} \mathrm{ha}^{-1}$ and this was $30.9 \%$ higher compared to non-AM rice. Total $\mathrm{K}$ uptake was significantly higher in AM-rice by $11.2 \%$. The interactive effect of rice varieties and AMF treatment was non-significant which is evident by the results that CR Dhan 205, CR Dhan 202 and Satyabhama did not show increase in total $\mathrm{K}$ uptake upon AMF treatment.

\section{Zinc ( $\mathrm{Zn}$ ) concentration and uptake as influenced by AMF treatment}

$\mathrm{Zn}$ concentration in grain and straw and its uptake was significantly affected upon AMF treatment (Table 7). Zn concentration in AM treated rice ranged from $26.44 \mathrm{mg} \mathrm{kg}^{-1}$ to 53.64 $\mathrm{mg} \mathrm{kg}{ }^{-1}$, this was higher by $32.4 \%$ compared to untreated rice. Maximum $\mathrm{Zn}$ accumulation was recorded for Satyabhama $\left(72.7 \mathrm{mg} \mathrm{kg}^{-1}\right)$ upon AMF colonization but in control plants, Pyari accumulated almost similar $\mathrm{Zn}$ content 
(71.2 $\mathrm{mg} \mathrm{kg}^{-1}$ ). Zn uptake by grain was $31.5 \%$ higher in AMF treated rice plants. Satyabhama was recorded for highest $\mathrm{Zn}$ uptake in grain $\left(214.56 \mathrm{~g} \mathrm{ha}^{-1}\right)$, in straw $\left(508.9 \mathrm{~g} \mathrm{ha}^{-1}\right)$ and their total $\left(723.46 \mathrm{~g} \mathrm{ha}^{-1}\right)$. A difference of only $10.8 \%$ was noted for total $\mathrm{Zn}$ uptake between AMF treated and control rice plants. The interaction between rice varieties and AMF treatment was found to be non-significant which is clear from the observation that seven out of ten rice varieties did not show increase in $\mathrm{Zn}$ concentration and uptake in straw. But the interaction was significant for $\mathrm{Zn}$ content in grain and straw and total $\mathrm{Zn}$ uptake.

Iron $(\mathrm{Fe})$ concentration and uptake as influenced by AMF treatment

Fe concentration in grain and straw was $24.6 \%$ and $22.1 \%$ higher in AMF treated rice (Table 8). Maximum $\mathrm{Fe}$ concentration in grain was recorded for Anjali (59.09 $\mathrm{mg} \mathrm{kg}^{-1}$ ) and lowest being for Vandana (36.8 $\mathrm{mg} \mathrm{kg}^{-1}$ ) after AMF treatment. The highest $\mathrm{Fe}$ accumulation in straw was exhibited by Satyabhama (354.79 $\mathrm{mg} \mathrm{kg}^{-1}$ ). The total $\mathrm{Fe}$ uptake in AMF treated rice plants ranged from $1584.63 \mathrm{~g}^{-1} \mathrm{ha}^{-1}$ to $2697.93 \mathrm{~g} \mathrm{ha}^{-1}$ which is almost $22.4 \%$ higher compared to untreated plants. The interactive effect of AMF and rice varieties was significant as all the rice varieties showed enhanced $\mathrm{Fe}$ content in grain and straw and its uptake by grain and straw and their total.

\section{Shoot dry weight and protein content in grain as influenced by AMF treatment}

Treatment with AMF significantly increased shoot dry weight of all the rice varieties under aerobic condition indicating a significant interaction between rice varieties and AMF treatment (Fig. 3). At 30 DAS, shoot dry weight was significantly higher in AMF treated by $57.5 \%$ compared to untreated control plants. A 54.9\% higher shoot dry weight was recorded for AMF treated rice plants compared to untreated control rice. Shoot dry weight among the AMF treated rice varieties ranged from $35.30 \mathrm{~g} \mathrm{~m}^{-2}$ to $88.67 \mathrm{~g} \mathrm{~m}^{-}$ ${ }^{2}$, being highest recorded for Satyabhama. AMF inoculation leads to $45 \%$ increase in protein content in grain (Fig. 4). Satyabhama had accumulated significantly higher protein content $(11.13 \%)$ over other rice varieties. Protein content among AMF treated rice ranged from $6.96 \%$ to $11.13 \%$. Lowest protein concentration was found in CR Dhan 201 (3.93\%) in control state.

\section{Correlation analyses}

Because maximum colonization of rice roots was observed at 30 DAS, we correlated percent colonization at 30 DAS and the plant parameters (Fig. 2). Non-significant positive correlation was exhibited by rice $\mathrm{N}$ and $\mathrm{K}$ uptake and percent colonization $\left(R^{2}=0.32, P=\right.$ 0.087 and $\left.R^{2}=0.11, P=0.336\right)$. The $R^{2}$ value for total $\mathrm{P}$ uptake and percent colonization was 0.755 , which depicts $75.5 \%$ variation in total $\mathrm{P}$ uptake of rice as explained by the fitted regression line. On the other hand, the $R^{2}$ value for total $\mathrm{Zn}$ and $\mathrm{Fe}$ uptake indicated $56.2 \%$ and $57.9 \%$ variability as explained by AMF treatment respectively.

Shoot dry weight was found to be significantly and positively correlated with percent colonization $\left(R^{2}=0.64, P=0.005\right)$. Regression analysis of total chlorophyll content and NR activity in AMF treated rice and percent colonization reveals $76.5 \%$ and $74.7 \%$ variability in total chlorophyll content and NR activity depicted by AMF treatment (Fig. 5). Aerobic rice is grown under non-puddled, non-flooded and non-saturated soil conditions (Bouman, 2001; Tuong and Bouman, 2003). The yield of rice under aerobic conditions heavily relies on the adequate supply of plant nutrients including $\mathrm{N}, \mathrm{P}, \mathrm{K}, \mathrm{Fe}, \mathrm{Zn}$ and others that may become deficient under aerobic conditions. 
Table.1 Characteristics of various rice genotypes used in the study

\begin{tabular}{|l|l|l|l|l|l|}
\hline S. & Genotypes & Adaptation & $\begin{array}{l}\text { Duration } \\
(\text { days })\end{array}$ & $\begin{array}{l}\text { Yield } \\
(\mathbf{t} / \mathbf{h a})\end{array}$ & Areas of adaptability \\
\hline 1. & Vandana & Upland & $90-95$ & 3.5 & Bihar, Jharkhand and Odisha \\
\hline 2. & CR Dhan 205 & Upland & $110-115$ & 4.2 & Tamilnadu, Odisha, Madhya Pradesh and Punjab \\
\hline 3. & CR Dhan 202 & Upland & $110-115$ & 3.7 & Jharkhand and Odisha \\
\hline 4. & Heera & Upland & $70-80$ & 3.0 & Jharkhand and Odisha \\
\hline 5. & Anjali & Upland & $90-100$ & 3.5 & Bihar, Jharkhand and Odisha \\
\hline 6. & CR Dhan 201 & Upland & $110-115$ & 3.8 & Bihar and Chattisgarh \\
\hline 7. & Pyari & Upland & $115-120$ & 4.2 & Bihar, Jharkhand and Odisha \\
\hline 8. & Satyabhama & Upland & $90-110$ & 4.0 & Bihar, Jharkhand and Odisha \\
\hline 9. & DhalaHeera & Upland & $75-80$ & 3.5 & Bihar, Jharkhand and Odisha \\
\hline 10. & CR Dhan 204 & Upland & $100-110$ & 4.0 & Jharkhand and Odisha \\
\hline
\end{tabular}

Table.2 Physical and chemical characteristics of initial soil samples

\begin{tabular}{|c|c|}
\hline Features & Range \\
\hline 1. Physical characteristics & \\
\hline Sand $(\%)$ & 52.31 \\
\hline Silt $(\%)$ & 25.25 \\
\hline Clay $(\%)$ & 25.49 \\
\hline 2. Chemical Characteristics & \\
\hline Soil organic carbon (\%) & 0.66 \\
\hline Soil Available $\mathbf{N}\left(\mathrm{kg} \mathrm{N} \mathrm{ha}^{-1}\right)$ & 148.35 \\
\hline Soil Available P (kg P ha $\left.{ }^{-1}\right)$ & 19.43 \\
\hline Soil Available K (kg K ha ${ }^{-1}$ ) & 239.53 \\
\hline Soil Fe (mgkg $\left.{ }^{-1}\right)$ & 11.52 \\
\hline Soil Zn $\left(\mathrm{mgkg}^{-1}\right)$ & 3.26 \\
\hline Soil pH (pHTestr 30 pH metre) & 7.4 \\
\hline Soil EC(ECTestr 11+)(mS/cm²) & 973 \\
\hline
\end{tabular}


Table.3 Interactive effect of rice varieties and AM colonization on nutrient concentration at flag leaf stage in rice ${ }^{\mathrm{a}}$

\begin{tabular}{|c|c|c|c|c|c|c|c|c|c|c|}
\hline & \multicolumn{2}{|c|}{ Shoot N (\%) } & \multicolumn{2}{|c|}{ Shoot P (\%) } & \multicolumn{2}{|c|}{ Shoot K (\%) } & \multicolumn{2}{|c|}{ Shoot Zn (mg kg $\left.{ }^{-1}\right)$} & \multicolumn{2}{|c|}{ Shoot Fe $\left(\mathrm{mg} \mathrm{kg}^{-1}\right)$} \\
\hline Varieties & $-\mathrm{AMF}$ & $+\mathrm{AMF}$ & $-\mathrm{AMF}$ & $+\mathrm{AMF}$ & $-\mathrm{AMF}$ & $+\mathrm{AMF}$ & $-\mathrm{AMF}$ & $+\mathrm{AMF}$ & $-\mathrm{AMF}$ & $+\mathrm{AMF}$ \\
\hline Vandana & $1.23 \mathrm{~cd}$ & $1.77 \mathrm{ab}$ & $0.09 \mathrm{c}$ & $0.15 b c$ & $1.01 \mathrm{~d}$ & $1.56 b c$ & $26.38 d$ & $36.46 \mathrm{~cd}$ & $43.42 \mathrm{def}$ & $53.65 \mathrm{de}$ \\
\hline $\begin{array}{l}\text { CR Dhan } \\
205\end{array}$ & $1.01 \mathrm{~d}$ & $1.53 \mathrm{bc}$ & $0.11 \mathrm{c}$ & $0.23 \mathrm{ab}$ & $0.99 d$ & $2.03 \mathrm{ab}$ & $30.52 \mathrm{~cd}$ & $40.6 \mathrm{bcd}$ & $33.82 \mathrm{ef}$ & $44.05 \mathrm{def}$ \\
\hline $\begin{array}{l}\text { CR Dhan } \\
202\end{array}$ & $0.99 \mathrm{~d}$ & $1.64 \mathrm{abc}$ & $0.10 \mathrm{c}$ & $0.24 \mathrm{ab}$ & $1.31 \mathrm{~cd}$ & $1.35 \mathrm{~cd}$ & $39.15 \mathrm{bcd}$ & 49.23abc & 45.49def & $55.72 \mathrm{de}$ \\
\hline Heera & $1.23 \mathrm{~cd}$ & $2.11 \mathrm{a}$ & $0.11 \mathrm{c}$ & $0.21 \mathrm{ab}$ & $1.11 \mathrm{~d}$ & $1.52 b c$ & $36.42 \mathrm{~cd}$ & $46.5 \mathrm{abc}$ & $33.29 \mathrm{ef}$ & 43.52def \\
\hline Anjali & $1.01 \mathrm{~d}$ & $1.85 \mathrm{ab}$ & $0.07 \mathrm{c}$ & $0.16 b c$ & $1.26 \mathrm{~cd}$ & $2.11 \mathrm{ab}$ & $42.58 \mathrm{bcd}$ & $52.66 \mathrm{ab}$ & $21.76 f$ & $31.99 \mathrm{ef}$ \\
\hline $\begin{array}{l}\text { CR Dhan } \\
201\end{array}$ & $0.97 d$ & $1.54 \mathrm{bc}$ & $0.11 \mathrm{c}$ & $0.15 b c$ & $1.03 d$ & $2.31 \mathrm{ab}$ & 29.25 & $39.33 \mathrm{bcd}$ & $84.29 \mathrm{~cd}$ & $94.52 c$ \\
\hline Pyari & $1.05 d$ & $2.11 \mathrm{a}$ & $0.09 \mathrm{c}$ & $0.27 \mathrm{ab}$ & $1.05 \mathrm{~d}$ & $2.43 a$ & $38.58 \mathrm{bcd}$ & 48.66abc & $62.02 \mathrm{de}$ & $72.25 d$ \\
\hline Satyabhama & $1.16 \mathrm{~d}$ & $2.17 \mathrm{a}$ & $0.08 \mathrm{c}$ & $0.30 \mathrm{a}$ & $1.13 d$ & $2.51 \mathrm{a}$ & $47.35 \mathrm{abc}$ & $57.43 \mathrm{a}$ & $149.56 a b$ & $159.79 a$ \\
\hline DhalaHeera & $0.89 d$ & $1.36 \mathrm{bcd}$ & $0.12 \mathrm{c}$ & $0.26 \mathrm{ab}$ & $1.09 \mathrm{~d}$ & $1.56 b c$ & $29.28 d$ & $39.36 \mathrm{bcd}$ & $84.82 \mathrm{~cd}$ & $95.05 c$ \\
\hline $\begin{array}{l}\text { CR Dhan } \\
204\end{array}$ & $1.12 \mathrm{~d}$ & $1.75 \mathrm{ab}$ & $0.09 \mathrm{c}$ & $0.19 a b c$ & $1.06 \mathrm{~d}$ & $1.43 \mathrm{bcd}$ & $35.32 \mathrm{~cd}$ & $45.4 \mathrm{bcd}$ & $121.62 b c$ & $131.85 b$ \\
\hline $\begin{array}{l}\text { Analysis of } \\
\text { Variance } \\
\end{array}$ & & & & & & & & & & \\
\hline Variety & & $0.0021 * * *$ & & $0.0005 * * *$ & & $0.0126^{*}$ & & $0.0069 * *$ & & $0.0012 * * *$ \\
\hline $\begin{array}{l}\text { Variety } \\
\times \mathbf{A M F}\end{array}$ & & $0.0121 *$ & & $0.0021 * *$ & & $0.0210^{*}$ & & $0.1002^{\mathrm{ns}}$ & & $0.0121 *$ \\
\hline
\end{tabular}

$*, * *, * * * * * * * \mathrm{~F}$ values significant at the $\mathrm{P}=0.05, \mathrm{P}=0.01, \mathrm{P}=0.001$ and $\mathrm{P} \leq 0.0001$ levels, respectively. ns means non-significant at the $\mathrm{P}=0.05$ level.

${ }^{a}$ Data are average of three replicates. Different letters indicate statistical significance at the $\mathrm{P}=0.05$ level within the same column and same conditions of cultivation. 
Table.4 Interactive effect of rice varieties and AM colonization on $\mathrm{N}$ concentration and uptake in rice ${ }^{\mathrm{a}}$

\begin{tabular}{|c|c|c|c|c|c|c|c|c|c|c|}
\hline \multirow[b]{2}{*}{ Varieties } & \multicolumn{2}{|c|}{$\begin{array}{c}\mathbf{N} \text { concentration in } \\
\text { grain }(\%)\end{array}$} & \multicolumn{2}{|c|}{$\begin{array}{l}\mathbf{N} \text { concentration } \\
\text { in straw }(\%)\end{array}$} & \multicolumn{2}{|c|}{$\begin{array}{c}\text { N uptake by grain } \\
\left(\mathrm{kgha}^{-1}\right)\end{array}$} & \multicolumn{2}{|c|}{$\begin{array}{c}\text { N uptake by straw } \\
\left(\mathrm{kgha}^{-1}\right)\end{array}$} & \multicolumn{2}{|c|}{$\begin{array}{c}\text { Total N uptake } \\
\left(\mathrm{kgha}^{-1}\right)\end{array}$} \\
\hline & $-\mathrm{AMF}$ & $+\mathrm{AMF}$ & $-\mathrm{AMF}$ & $+\mathrm{AMF}$ & $-\mathrm{AMF}$ & $+\mathrm{AMF}$ & $-\mathrm{AMF}$ & $+\mathrm{AMF}$ & $-\mathrm{AMF}$ & $+\mathrm{AMF}$ \\
\hline Vandana & $1.30 \mathrm{bc}$ & $1.61 \mathrm{abc}$ & $0.41 b c$ & $0.54 \mathrm{abc}$ & $45.50 \mathrm{~cd}$ & $56.35 b c$ & $26.65 \mathrm{bcd}$ & $35.10 \mathrm{bc}$ & $72.15 \mathrm{bcd}$ & $91.45 \mathrm{ab}$ \\
\hline $\begin{array}{l}\text { CR Dhan } \\
205\end{array}$ & $1.25 b c$ & $1.76 a b$ & $0.48 b c$ & $0.55 \mathrm{abc}$ & $52.50 \mathrm{bcd}$ & 73.92ab & $34.56 b c$ & $39.60 b c$ & $87.06 b c$ & $113.52 \mathrm{a}$ \\
\hline $\begin{array}{l}\text { CR Dhan } \\
202 \\
\end{array}$ & $1.16 \mathrm{c}$ & $1.34 \mathrm{bc}$ & $0.39 \mathrm{bcd}$ & $0.59 \mathrm{abc}$ & $42.92 \mathrm{~cd}$ & $49.58 \mathrm{bcd}$ & $26.13 \mathrm{bcd}$ & $39.53 b c$ & $69.05 \mathrm{bcd}$ & $89.11 b c$ \\
\hline Heera & $1.22 b c$ & $1.73 \mathrm{ab}$ & $0.44 b c$ & $0.59 \mathrm{abc}$ & $36.60 \mathrm{cde}$ & $51.90 \mathrm{bcd}$ & $26.40 \mathrm{bcd}$ & $35.40 \mathrm{bc}$ & $63.00 \mathrm{~cd}$ & $87.30 b c$ \\
\hline Anjali & $0.80 \mathrm{~cd}$ & $1.66 a b c$ & $0.24 \mathrm{~cd}$ & $0.59 \mathrm{abc}$ & $28.00 \mathrm{de}$ & $58.10 b c$ & $15.60 \mathrm{~cd}$ & $38.35 b c$ & $43.60 \mathrm{cde}$ & $96.45 \mathrm{ab}$ \\
\hline $\begin{array}{l}\text { CR Dhan } \\
201\end{array}$ & $0.66 \mathrm{~cd}$ & $1.17 \mathrm{c}$ & $0.35 \mathrm{bcd}$ & $0.61 \mathrm{ab}$ & $25.08 \mathrm{de}$ & $44.46 \mathrm{~cd}$ & $23.80 \mathrm{bcd}$ & 41.48ab & $48.88 \mathrm{cde}$ & $85.94 b c$ \\
\hline Pyari & $1.17 b c$ & $1.75 \mathrm{ab}$ & $0.46 b c$ & $0.62 \mathrm{a}$ & $49.14 \mathrm{bcd}$ & $73.50 \mathrm{ab}$ & $33.12 b c$ & $44.64 a$ & $82.26 b c$ & $118.14 \mathrm{a}$ \\
\hline Satyabhama & $1.70 \mathrm{ab}$ & $1.87 \mathrm{a}$ & $0.61 \mathrm{ab}$ & $0.61 \mathrm{ab}$ & $68.00 \mathrm{ab}$ & $74.80 \mathrm{a}$ & $42.70 \mathrm{ab}$ & $42.70 \mathrm{ab}$ & $110.70 \mathrm{a}$ & $117.50 \mathrm{a}$ \\
\hline DhalaHeera & $0.93 \mathrm{~cd}$ & $1.68 \mathrm{abc}$ & $0.45 b c$ & $0.60 \mathrm{ab}$ & $32.55 \mathrm{cde}$ & $58.80 \mathrm{bc}$ & $29.25 \mathrm{bcd}$ & $39.00 b c$ & $61.80 \mathrm{~cd}$ & $97.80 \mathrm{ab}$ \\
\hline $\begin{array}{l}\text { CR Dhan } \\
204 \\
\end{array}$ & $0.77 \mathrm{~cd}$ & $1.28 b c$ & $0.35 \mathrm{bcd}$ & $0.51 \mathrm{abc}$ & $30.80 \mathrm{cde}$ & $51.20 \mathrm{bcd}$ & $24.50 \mathrm{bcd}$ & $35.70 b c$ & $55.30 \mathrm{~cd}$ & $86.90 b c$ \\
\hline \multicolumn{11}{|l|}{$\begin{array}{l}\text { Analysis of } \\
\text { Variance }\end{array}$} \\
\hline Variety & & $0.0121 * *$ & & $0.6301^{\mathrm{ns}}$ & & $0.0326^{*}$ & & $0.0169 * *$ & & $0.0012 * * *$ \\
\hline $\begin{array}{l}\text { Variety } \\
\times \mathbf{A M F}\end{array}$ & & $0.0421 *$ & & $0.5023^{\mathrm{ns}}$ & & $0.0310^{*}$ & & $0.1102^{\mathrm{ns}}$ & & $0.0121 * *$ \\
\hline
\end{tabular}

$*, * *, * *, * * * * \mathrm{~F}$ values significant at the $\mathrm{P}=0.05, \mathrm{P}=0.01, \mathrm{P}=0.001$ and $\mathrm{P} \leq 0.0001$ levels, respectively. ns means non-significant at the $\mathrm{P}=0.05$ level.

${ }^{a}$ Data are average of three replicates. Different letters indicate statistical significance at the $\mathrm{P}=0.05$ level within the same column and same conditions of cultivation. 
Table.5 Interactive effect of rice varieties and AM colonization on $\mathrm{P}$ concentration and uptake in rice ${ }^{\mathrm{a}}$

\begin{tabular}{|c|c|c|c|c|c|c|c|c|c|c|}
\hline \multirow[b]{2}{*}{ Varieties } & \multicolumn{2}{|c|}{$\begin{array}{c}\text { P concentration in } \\
\text { grain } \\
(\%)\end{array}$} & \multicolumn{2}{|c|}{$\begin{array}{c}\text { P concentration in } \\
\text { straw } \\
(\%)\end{array}$} & \multicolumn{2}{|c|}{$\begin{array}{c}\text { P uptake by grain } \\
\left(\mathrm{kgha}^{-1}\right)\end{array}$} & \multicolumn{2}{|c|}{$\begin{array}{c}\text { P uptake by straw } \\
\left(\mathrm{kgha}^{-1}\right)\end{array}$} & \multicolumn{2}{|c|}{$\begin{array}{l}\text { Total P uptake } \\
\text { (kgha-1) }\end{array}$} \\
\hline & $-\mathrm{AMF}$ & $+\mathrm{AMF}$ & $-A M F$ & $+\mathrm{AMF}$ & $-\mathrm{AMF}$ & $+\mathrm{AMF}$ & -AMF & $+\mathrm{AMF}$ & $-\mathrm{AMF}$ & $+\mathrm{AMF}$ \\
\hline Vandana & $0.16 \mathrm{bcd}$ & $0.19 \mathrm{bcd}$ & $0.14 b$ & $0.19 \mathrm{ab}$ & $5.60 \mathrm{~cd}$ & $6.65 \mathrm{~cd}$ & $9.10 b c$ & $12.35 \mathrm{abc}$ & $14.70 \mathrm{c}$ & $19.00 \mathrm{bc}$ \\
\hline $\begin{array}{l}\text { CR Dhan } \\
205\end{array}$ & $0.18 \mathrm{bcd}$ & $0.21 b c$ & $0.09 c$ & $0.14 b$ & $7.56 \mathrm{bcd}$ & $8.82 b c$ & $6.48 c$ & $10.08 \mathrm{bc}$ & $14.04 \mathrm{c}$ & $18.90 b c$ \\
\hline $\begin{array}{l}\text { CR Dhan } \\
202 \\
\end{array}$ & $0.15 \mathrm{~cd}$ & $0.22 b c$ & $0.13 b$ & $0.18 \mathrm{ab}$ & $5.55 \mathrm{~cd}$ & $8.14 b c$ & $8.71 b c$ & $12.06 \mathrm{abc}$ & $14.26 \mathrm{c}$ & $20.20 a b c$ \\
\hline Heera & $0.17 \mathrm{bcd}$ & $0.17 \mathrm{bcd}$ & $0.14 b$ & $0.19 \mathrm{ab}$ & $5.10 \mathrm{~cd}$ & $5.10 \mathrm{~cd}$ & $8.40 \mathrm{bc}$ & $11.40 \mathrm{abc}$ & $13.50 \mathrm{c}$ & $16.50 \mathrm{bc}$ \\
\hline Anjali & $0.16 \mathrm{~cd}$ & $0.22 b c$ & $0.15 b$ & $0.20 \mathrm{a}$ & $5.60 \mathrm{~cd}$ & $7.70 \mathrm{bcd}$ & $9.75 b c$ & $13.00 \mathrm{abc}$ & $15.35 b c$ & $20.70 \mathrm{abc}$ \\
\hline $\begin{array}{l}\text { CR Dhan } \\
201\end{array}$ & $0.17 \mathrm{bcd}$ & $0.24 b c$ & $0.18 \mathrm{ab}$ & $0.23 a$ & $6.46 \mathrm{~cd}$ & $9.12 \mathrm{abc}$ & $12.24 \mathrm{abc}$ & $15.64 a b$ & $18.70 b c$ & $24.76 a b$ \\
\hline Pyari & $0.16 \mathrm{~cd}$ & $0.25 b c$ & $0.12 b c$ & $0.17 \mathrm{ab}$ & $6.72 \mathrm{~cd}$ & $10.50 \mathrm{ab}$ & $8.64 b c$ & $12.24 \mathrm{abc}$ & $15.36 b c$ & $22.74 \mathrm{ab}$ \\
\hline Satyabhama & $0.21 b c$ & $0.32 \mathrm{a}$ & $0.19 a b$ & $0.24 \mathrm{a}$ & $8.40 b c$ & $12.80 \mathrm{a}$ & $13.30 \mathrm{abc}$ & $16.80 \mathrm{a}$ & $21.70 \mathrm{abc}$ & $29.60 \mathrm{a}$ \\
\hline DhalaHeera & $0.19 \mathrm{bcd}$ & $0.22 b c$ & $0.14 b$ & $0.19 \mathrm{ab}$ & $6.65 \mathrm{~cd}$ & $7.70 \mathrm{bcd}$ & $9.10 b c$ & $12.35 \mathrm{abc}$ & $15.75 b c$ & $20.05 \mathrm{abc}$ \\
\hline $\begin{array}{l}\text { CR Dhan } \\
204\end{array}$ & $0.15 \mathrm{~cd}$ & $0.28 \mathrm{ab}$ & $0.16 a b$ & $0.16 a b$ & $6.00 \mathrm{~cd}$ & $11.20 \mathrm{ab}$ & 11.20abc & 11.20abc & $17.20 \mathrm{bc}$ & $22.40 \mathrm{ab}$ \\
\hline $\begin{array}{l}\text { Analysis of } \\
\text { Variance } \\
\end{array}$ & & & & & & & & & & \\
\hline Variety & & $0.0123 * * *$ & & $0.0001 * * * *$ & & $0.0291 *$ & & $0.2131^{\mathrm{ns}}$ & & $0.0251^{*}$ \\
\hline $\begin{array}{l}\text { Variety } \\
\times \text { AMIF }\end{array}$ & & $0.0012 * *$ & & $0.0081 * *$ & & $0.0010 * * *$ & & $0.0351 *$ & & $0.0023 * *$ \\
\hline
\end{tabular}

$*, * *, * * *, * * * F$ values significant at the $\mathrm{P}=0.05, \mathrm{P}=0.01, \mathrm{P}=0.001$ and $\mathrm{P} \leq 0.0001$ levels, respectively. $\mathrm{ns}$ means non-significant at the $\mathrm{P}=0.05$ level.

${ }^{a}$ Data are average of three replicates. Different letters indicate statistical significance at the $\mathrm{P}=0.05$ level within the same column and same conditions of cultivation. 
Table.6 Interactive effect of rice varieties and AM colonization on $\mathrm{K}$ concentration and uptake in rice ${ }^{\mathrm{a}}$

\begin{tabular}{|c|c|c|c|c|c|c|c|c|c|c|}
\hline \multirow[b]{2}{*}{ Varieties } & \multicolumn{2}{|c|}{$\begin{array}{l}\text { K concentration } \\
\text { in grain }(\%)\end{array}$} & \multicolumn{2}{|c|}{$\begin{array}{l}\text { K concentration } \\
\text { in straw }(\%)\end{array}$} & \multicolumn{2}{|c|}{$\begin{array}{c}\text { K uptake by grain } \\
\left(\mathrm{kgha}^{-1}\right)\end{array}$} & \multicolumn{2}{|c|}{$\begin{array}{c}\text { K uptake by } \\
\text { straw } \\
\left(\mathrm{kgha}^{-1}\right)\end{array}$} & \multicolumn{2}{|c|}{$\begin{array}{l}\text { Total K uptake } \\
\qquad\left(\mathrm{kgha}^{-1}\right)\end{array}$} \\
\hline & -AMF & $+\mathrm{AMF}$ & $-\mathrm{AMF}$ & $+\mathrm{AMF}$ & $-\mathrm{AMF}$ & $+\mathrm{AMF}$ & -AMF & $+\mathrm{AMF}$ & -AMF & $+\mathrm{AMF}$ \\
\hline Vandana & $0.35 \mathrm{a}$ & $0.38 \mathrm{a}$ & $1.18 b c$ & 1.49ab & $12.25 b c$ & $13.30 b c$ & $76.70 \mathrm{bc}$ & $96.85 \mathrm{ab}$ & $88.95 b c$ & $110.15 \mathrm{abc}$ \\
\hline $\begin{array}{l}\text { CR Dhan } \\
205 \\
\end{array}$ & $0.32 \mathrm{a}$ & $0.43 a$ & $1.20 \mathrm{bc}$ & $0.96 b c$ & $13.44 b c$ & $18.06 \mathrm{ab}$ & $86.40 \mathrm{abc}$ & $69.12 b c$ & $99.84 a b c$ & $87.18 b c$ \\
\hline $\begin{array}{l}\text { CR Dhan } \\
202 \\
\end{array}$ & $0.35 \mathrm{a}$ & $0.47 \mathrm{a}$ & $0.93 b c$ & $0.79 b c$ & $12.95 b c$ & $17.39 \mathrm{abc}$ & $62.31 b c$ & $52.93 \mathrm{c}$ & $75.26 \mathrm{c}$ & $70.32 c$ \\
\hline Heera & $0.38 \mathrm{a}$ & $0.47 \mathrm{a}$ & $0.94 b c$ & $1.12 b c$ & $11.40 b c$ & $14.10 \mathrm{bc}$ & $56.40 c$ & $67.20 \mathrm{bc}$ & $67.80 c$ & $81.3 \mathrm{bc}$ \\
\hline Anjali & $0.27 \mathrm{ab}$ & $0.51 \mathrm{a}$ & $1.17 b c$ & $1.60 \mathrm{ab}$ & $9.45 c$ & $17.85 \mathrm{abc}$ & $76.05 \mathrm{bc}$ & $104.00 \mathrm{a}$ & $85.50 b c$ & $121.85 \mathrm{ab}$ \\
\hline $\begin{array}{l}\text { CR Dhan } \\
201\end{array}$ & $0.29 \mathrm{ab}$ & $0.49 a$ & $1.29 b c$ & $1.53 \mathrm{ab}$ & $11.02 b c$ & $18.62 \mathrm{ab}$ & $87.72 \mathrm{abc}$ & $104.04 a$ & $98.74 \mathrm{abc}$ & $122.66 \mathrm{ab}$ \\
\hline Pyari & $0.39 a$ & $0.46 a$ & $1.17 \mathrm{bc}$ & $1.39 \mathrm{abc}$ & $16.38 \mathrm{abc}$ & $19.32 \mathrm{ab}$ & $84.24 \mathrm{abc}$ & $100.08 \mathrm{a}$ & 100.62 & $119.4 a b c$ \\
\hline Satyabhama & $0.53 \mathrm{a}$ & $0.55 \mathrm{a}$ & $1.58 \mathrm{ab}$ & $1.39 \mathrm{abc}$ & $21.20 \mathrm{ab}$ & $22.00 \mathrm{a}$ & $110.60 \mathrm{a}$ & $97.30 \mathrm{ab}$ & $131.80 \mathrm{a}$ & $119.3 \mathrm{abc}$ \\
\hline DhalaHeera & $0.33 \mathrm{a}$ & $0.52 \mathrm{a}$ & $1.15 b c$ & $1.62 \mathrm{a}$ & $11.55 b c$ & $18.20 \mathrm{ab}$ & $107.25 \mathrm{a}$ & $107.25 \mathrm{a}$ & $118.80 \mathrm{abc}$ & $125.45 \mathrm{ab}$ \\
\hline $\begin{array}{l}\text { CR Dhan } \\
204\end{array}$ & $0.37 \mathrm{a}$ & $0.43 a$ & $1.28 \mathrm{bc}$ & $1.51 \mathrm{ab}$ & $14.80 \mathrm{bc}$ & $17.20 \mathrm{abc}$ & 89.60abc & $105.70 \mathrm{a}$ & 104.40abc & $122.9 \mathrm{ab}$ \\
\hline $\begin{array}{l}\text { Analysis of } \\
\text { Variance } \\
\end{array}$ & & & & & & & & & & \\
\hline Variety & & $0.1352^{\mathrm{ns}}$ & & $0.0014 * *$ & & $0.0051 * *$ & & $0.0151 *$ & & $0.0211 *$ \\
\hline $\begin{array}{l}\text { Variety } \\
\times \mathbf{A M I F}\end{array}$ & & $0.4251^{\mathrm{ns}}$ & & $0.0123 *$ & & $0.0211 *$ & & $0.0032 *$ & & $0.1238^{\mathrm{ns}}$ \\
\hline
\end{tabular}

$*, * *, * * *, * * * * \mathrm{~F}$ values significant at the $\mathrm{P}=0.05, \mathrm{P}=0.01, \mathrm{P}=0.001$ and $\mathrm{P} \leq 0.0001$ levels, respectively. $\mathrm{ns}$ means non-significant at the $\mathrm{P}=0.05$ level. ${ }^{a}$ Data are average of three replicates. Different letters indicate statistical significance at the $\mathrm{P}=0.05$ level within the same column and same conditions of cultivation. 
Table.7 Interactive effect of rice varieties and AM colonization on $\mathrm{Zn}$ concentration and uptake in rice ${ }^{\mathrm{a}}$

\begin{tabular}{|c|c|c|c|c|c|c|c|c|c|c|}
\hline \multirow[b]{2}{*}{ Varieties } & \multicolumn{2}{|c|}{$\begin{array}{l}\text { Zn concentration } \\
\text { in grain }\left(\mathrm{mgkg}^{-1}\right)\end{array}$} & \multicolumn{2}{|c|}{$\begin{array}{l}\text { Zn concentration } \\
\text { in straw }\left(\mathrm{mgkg}^{-1}\right)\end{array}$} & \multicolumn{2}{|c|}{$\begin{array}{l}\text { Zn uptake by grain } \\
\left(\text { gha }^{-1}\right)\end{array}$} & \multicolumn{2}{|c|}{$\begin{array}{c}\text { Zn uptake by straw } \\
\left(\text { gha }^{-1}\right)\end{array}$} & \multicolumn{2}{|c|}{$\begin{array}{l}\text { Total Zn uptake } \\
\left(\mathrm{gha}^{-1}\right)\end{array}$} \\
\hline & -AMF & $+\mathrm{AMF}$ & $-\mathrm{AMF}$ & $+\mathrm{AMF}$ & -AMF & $+\mathrm{AMF}$ & -AMF & $+\mathrm{AMF}$ & -AMF & $+\mathrm{AMF}$ \\
\hline Vandana & $33.60 \mathrm{bcd}$ & $34.11 \mathrm{bcd}$ & $48.77 \mathrm{c}$ & $51.73 b c$ & $117.60 \mathrm{~cd}$ & $119.38 \mathrm{~cd}$ & $317.01 \mathrm{~d}$ & $336.24 d$ & $434.61 d$ & $455.63 \mathrm{~d}$ \\
\hline $\begin{array}{l}\text { CR Dhan } \\
205\end{array}$ & $19.27 \mathrm{~cd}$ & $26.44 \mathrm{~cd}$ & $55.30 b c$ & $55.87 b c$ & $80.93 d$ & $111.05 \mathrm{~cd}$ & $398.16 \mathrm{~cd}$ & $402.26 \mathrm{bcd}$ & $479.09 \mathrm{~d}$ & 513.31cd \\
\hline $\begin{array}{l}\text { CR Dhan } \\
202\end{array}$ & $35.20 \mathrm{bcd}$ & $49.04 a b c$ & $63.77 \mathrm{ab}$ & $64.50 \mathrm{ab}$ & $130.24 \mathrm{bcd}$ & $181.45 \mathrm{ab}$ & $427.25 b c$ & $432.15 b c$ & $557.49 \mathrm{bcd}$ & $613.59 b c$ \\
\hline Heera & $26.60 \mathrm{~cd}$ & $49.51 \mathrm{abc}$ & $60.60 \mathrm{abc}$ & $61.77 \mathrm{abc}$ & $79.80 \mathrm{~d}$ & $148.53 b c$ & $363.60 \mathrm{~cd}$ & $370.62 \mathrm{~cd}$ & $443.40 \mathrm{~d}$ & $519.15 \mathrm{~cd}$ \\
\hline Anjali & $39.40 \mathrm{bc}$ & $53.24 \mathrm{a}$ & $67.63 \mathrm{ab}$ & $67.93 \mathrm{ab}$ & $137.90 \mathrm{bc}$ & $186.34 \mathrm{ab}$ & $439.59 \mathrm{bc}$ & $441.54 b$ & $577.49 \mathrm{bcd}$ & $627.88 b$ \\
\hline $\begin{array}{l}\text { CR Dhan } \\
201 \\
\end{array}$ & $38.13 b c$ & $38.64 b c$ & $52.47 b c$ & $54.60 b c$ & $144.89 b c$ & $146.83 b c$ & $356.79 \mathrm{~cd}$ & $371.28 \mathrm{~cd}$ & $501.69 \mathrm{~cd}$ & $518.11 \mathrm{~cd}$ \\
\hline Pyari & $26.07 \mathrm{~cd}$ & $26.58 \mathrm{~cd}$ & $71.20 \mathrm{a}$ & 63.93abc & $109.49 \mathrm{~cd}$ & $111.64 \mathrm{~cd}$ & $460.29 b$ & $512.64 \mathrm{a}$ & $622.13 b$ & $571.93 \mathrm{bcd}$ \\
\hline Satyabhama & $52.40 \mathrm{ab}$ & $53.64 a$ & $62.47 \mathrm{abc}$ & $72.70 \mathrm{a}$ & $209.60 \mathrm{ab}$ & $214.56 a$ & $437.29 b c$ & $508.90 \mathrm{a}$ & $646.89 b$ & $723.46 a$ \\
\hline DhalaHeera & $33.20 \mathrm{bcd}$ & $47.04 \mathrm{abc}$ & $54.00 \mathrm{bc}$ & $54.63 b c$ & $116.20 \mathrm{~cd}$ & $164.64 \mathrm{abc}$ & $351.00 \mathrm{~cd}$ & $355.09 \mathrm{~cd}$ & $467.20 \mathrm{~d}$ & $519.73 \mathrm{~cd}$ \\
\hline $\begin{array}{l}\text { CR Dhan } \\
204\end{array}$ & $19.87 \mathrm{~cd}$ & $50.44 \mathrm{ab}$ & $60.20 \mathrm{abc}$ & $60.67 \mathrm{abc}$ & $79.48 d$ & 201.76ab & $421.40 \mathrm{bc}$ & $424.69 b c$ & $500.88 \mathrm{~cd}$ & $626.45 b$ \\
\hline \multicolumn{11}{|l|}{$\begin{array}{l}\text { Analysis of } \\
\text { Variance } \\
\end{array}$} \\
\hline Variety & & $0.0022 * *$ & & $0.0321 *$ & & $0.0035 * *$ & & $0.0029 * *$ & & $0.0215^{*}$ \\
\hline $\begin{array}{l}\text { Variety } \\
\times \text { AMF }\end{array}$ & & $0.0231 *$ & & $0.3351^{\mathrm{ns}}$ & & $0.0012 * *$ & & $0.2351^{\mathrm{ns}}$ & & $0.2211^{\mathrm{ns}}$ \\
\hline
\end{tabular}


Table.8 Interactive effect of rice varieties and AM colonization on Fe concentration and uptake in rice ${ }^{\mathrm{a}}$

\begin{tabular}{|c|c|c|c|c|c|c|c|c|c|c|}
\hline \multirow[b]{2}{*}{ Varieties } & \multicolumn{2}{|c|}{$\begin{array}{l}\text { Fe concentration in } \\
\text { grain }\left(\mathrm{mgkg}^{-1}\right)\end{array}$} & \multicolumn{2}{|c|}{$\begin{array}{l}\text { Fe concentration in } \\
\text { straw }\left(\mathrm{mgkg}^{-1}\right)\end{array}$} & \multicolumn{2}{|c|}{$\begin{array}{l}\text { Fe uptake by grain } \\
\qquad\left(\mathrm{gha}^{-1}\right)\end{array}$} & \multicolumn{2}{|c|}{$\begin{array}{l}\text { Fe uptake by straw } \\
\qquad\left(\mathrm{gha}^{-1}\right)\end{array}$} & \multicolumn{2}{|c|}{$\begin{array}{l}\text { Total Fe uptake } \\
\left(\mathrm{gha}^{-1}\right)\end{array}$} \\
\hline & -AMF & $+\mathrm{AMF}$ & $-\mathrm{AMF}$ & $+\mathrm{AMF}$ & $-\mathrm{AMF}$ & $+\mathrm{AMF}$ & $-\mathrm{AMF}$ & $+\mathrm{AMF}$ & $-\mathrm{AMF}$ & $+\mathrm{AMF}$ \\
\hline Vandana & $13.59 \mathrm{~d}$ & $36.80 \mathrm{~cd}$ & $148.33 d$ & $248.65 \mathrm{~cd}$ & $47.56 \mathrm{~d}$ & $128.80 \mathrm{~cd}$ & $964.14 \mathrm{e}$ & 1616.22cde & $1011.71 \mathrm{e}$ & $1745.02 \mathrm{~cd}$ \\
\hline $\begin{array}{l}\text { CR Dhan } \\
205\end{array}$ & $54.55 \mathrm{ab}$ & $54.42 \mathrm{ab}$ & $148.33 d$ & $239.05 \mathrm{~cd}$ & $229.11 \mathrm{a}$ & $228.56 a$ & $1067.97 \mathrm{e}$ & 1721.16bcd & $1297.08 \mathrm{e}$ & $1949.72 b c d$ \\
\hline $\begin{array}{l}\text { CR Dhan } \\
202 \\
\end{array}$ & $20.64 d$ & $47.19 \mathrm{bcd}$ & $250.40 \mathrm{bcd}$ & $250.72 \mathrm{bcd}$ & $76.36 \mathrm{~d}$ & $174.61 b c$ & $1677.68 \mathrm{~cd}$ & $1679.82 \mathrm{~cd}$ & $1754.05 \mathrm{~cd}$ & $1854.43 \mathrm{bcd}$ \\
\hline Heera & $51.30 \mathrm{abc}$ & $51.17 \mathrm{abc}$ & $143.20 \mathrm{~d}$ & $238.52 \mathrm{~cd}$ & $153.90 \mathrm{bcd}$ & $153.51 \mathrm{bcd}$ & $859.20 \mathrm{e}$ & 1431.12 de & $1013.10 \mathrm{e}$ & $1584.63 \mathrm{cde}$ \\
\hline Anjali & $55.89 \mathrm{ab}$ & $59.09 a$ & $226.67 \mathrm{~cd}$ & $226.99 \mathrm{~cd}$ & 195.61abc & $206.81 \mathrm{ab}$ & $1473.35 \mathrm{de}$ & $1475.43 \mathrm{de}$ & $1668.97 \mathrm{cde}$ & $1682.25 \mathrm{cde}$ \\
\hline $\begin{array}{l}\text { CR Dhan } \\
201\end{array}$ & $40.09 \mathrm{~cd}$ & $46.62 \mathrm{bcd}$ & $269.20 \mathrm{bcd}$ & $289.52 b c$ & $152.34 \mathrm{bcd}$ & $177.15 b c$ & $1830.56 b c$ & $1968.73 \mathrm{abc}$ & $1982.90 \mathrm{bcd}$ & $2145.89 b c$ \\
\hline Pyari & $23.37 d$ & $49.91 b c$ & $200.27 \mathrm{~cd}$ & $267.25 \mathrm{bcd}$ & $98.15 d$ & $209.62 \mathrm{ab}$ & 1441.94de & $1924.20 \mathrm{abc}$ & 1540.09 cde & $2133.82 b c$ \\
\hline Satyabhama & $43.73 \mathrm{~cd}$ & $53.60 \mathrm{ab}$ & $293.20 b c$ & $354.79 a$ & $174.92 \mathrm{bc}$ & $214.41 \mathrm{ab}$ & $2052.40 \mathrm{ab}$ & $2483.53 a$ & $2227.32 b$ & $2697.93 \mathrm{a}$ \\
\hline DhalaHeera & $48.88 \mathrm{bcd}$ & $55.41 \mathrm{ab}$ & $289.73 b c$ & $290.05 b c$ & $171.08 \mathrm{bc}$ & $193.93 \mathrm{abc}$ & $1883.24 b c$ & $1885.32 b c$ & $2054.32 b c$ & $2079.26 b c$ \\
\hline $\begin{array}{l}\text { CR Dhan } \\
204\end{array}$ & $47.92 \mathrm{bcd}$ & $44.46 \mathrm{~cd}$ & $268.27 \mathrm{bcd}$ & $326.85 a b$ & $191.68 \mathrm{abc}$ & $177.84 b c$ & $1877.89 b c$ & $2287.95 \mathrm{a}$ & $2069.57 b c$ & $2465.79 b$ \\
\hline \multicolumn{11}{|l|}{$\begin{array}{l}\text { Analysis of } \\
\text { Variance }\end{array}$} \\
\hline Variety & & $0.0008 * * *$ & & $0.0054 * *$ & & $0.0008 * * *$ & & $0.0043 * *$ & & $0.0212 *$ \\
\hline $\begin{array}{l}\text { Variety } \\
\times \text { AMF }\end{array}$ & & $0.0023 * *$ & & $0.2190^{\mathrm{ns}}$ & & $0.0012 * *$ & & $0.1320^{\mathrm{ns}}$ & & $0.2135^{\mathrm{ns}}$ \\
\hline
\end{tabular}


Table.9 Interactive effect of rice varieties and AM colonization on chlorophyll content and NR activity of rice ${ }^{\mathrm{a}}$

\begin{tabular}{|c|c|c|c|c|c|c|c|c|}
\hline & \multicolumn{2}{|c|}{$\begin{array}{l}\text { Chlorophyll a } \\
\text { (mg g } \text { fresh }^{-1} \text { ) } \\
\text { weight) }\end{array}$} & \multicolumn{2}{|c|}{$\begin{array}{l}\text { Chlorophyll b (mg } \\
\mathrm{g}^{-1} \text { fresh weight) }\end{array}$} & \multicolumn{2}{|c|}{$\begin{array}{l}\text { Total chlorophyll } \\
\text { (mg g }{ }^{-1} \text { fresh } \\
\text { weight) }\end{array}$} & \multicolumn{2}{|c|}{$\begin{array}{l}\text { Nitrate reductase ( } \mu \text { moles } \\
\text { of nitrite formed } h^{-1} g^{-1} \\
\text { fresh weight) }\end{array}$} \\
\hline Varieties & $-\mathrm{AMF}$ & $+\mathrm{AMF}$ & $-\mathrm{AMF}$ & $+\mathrm{AMF}$ & $-\mathrm{AMF}$ & $+\mathrm{AMF}$ & $-\mathrm{AMF}$ & $+\mathrm{AMF}$ \\
\hline Vandana & $0.77 \mathrm{~d}$ & $1.01 \mathrm{~cd}$ & $0.41 \mathrm{~cd}$ & $0.62 b c$ & $1.18 \mathrm{e}$ & $1.64 \mathrm{c}$ & $15.73 d$ & $31.70 b c$ \\
\hline $\begin{array}{l}\text { CR Dhan } \\
205\end{array}$ & $0.73 d$ & $1.80 \mathrm{~b}$ & $0.33 d$ & $0.58 \mathrm{bcd}$ & $1.06 \mathrm{e}$ & $2.38 \mathrm{ab}$ & $14.42 d$ & $33.00 b c$ \\
\hline $\begin{array}{l}\text { CR Dhan } \\
202\end{array}$ & $0.81 \mathrm{~d}$ & $1.41 \mathrm{c}$ & $0.55 \mathrm{bcd}$ & $0.66 b c$ & $1.36 \mathrm{de}$ & $2.07 \mathrm{~b}$ & $14.44 d$ & $32.92 b c$ \\
\hline Heera & $0.72 d$ & $1.08 \mathrm{~cd}$ & $0.52 \mathrm{bcd}$ & $0.57 \mathrm{bcd}$ & $1.24 \mathrm{e}$ & $1.66 \mathrm{c}$ & $15.05 d$ & $25.80 \mathrm{~cd}$ \\
\hline Anjali & $0.83 d$ & $1.34 \mathrm{c}$ & $0.42 \mathrm{~cd}$ & $0.68 b c$ & $1.25 \mathrm{e}$ & $2.02 \mathrm{~b}$ & $14.67 d$ & $30.89 \mathrm{bcd}$ \\
\hline $\begin{array}{l}\text { CR Dhan } \\
201\end{array}$ & $0.84 d$ & $1.24 \mathrm{c}$ & $0.45 \mathrm{~cd}$ & $0.69 b c$ & $1.29 \mathrm{e}$ & $1.93 b c$ & $14.33 d$ & $35.55 b$ \\
\hline Pyari & $0.90 \mathrm{~d}$ & $1.17 \mathrm{~cd}$ & $0.42 \mathrm{~cd}$ & $0.75 a$ & $1.32 \mathrm{de}$ & $1.92 b c$ & $14.59 d$ & $33.14 b c$ \\
\hline Satyabhama & $1.03 \mathrm{~cd}$ & $2.38 \mathrm{a}$ & $0.42 \mathrm{~cd}$ & $0.88 \mathrm{a}$ & $1.45 d$ & $3.26 \mathrm{a}$ & $14.58 \mathrm{~d}$ & $41.12 \mathrm{a}$ \\
\hline DhalaHeera & $0.84 d$ & $1.34 \mathrm{c}$ & $0.38 \mathrm{~cd}$ & $0.69 b c$ & $1.22 \mathrm{e}$ & $2.03 b$ & $14.73 d$ & $33.09 b c$ \\
\hline $\begin{array}{l}\text { CR Dhan } \\
204\end{array}$ & $0.82 \mathrm{~d}$ & $1.29 c$ & $0.35 d$ & $0.67 b c$ & $1.17 \mathrm{e}$ & $1.96 b c$ & $16.40 d$ & $25.25 \mathrm{~cd}$ \\
\hline \multicolumn{9}{|c|}{ Analysis of Variance } \\
\hline Variety & & $0.0125^{*}$ & & $0.0014 * *$ & & $0.0289 *$ & $* *$ & $0.0024 * *$ \\
\hline $\begin{array}{l}\text { Variety } \\
\times \text { Condition }\end{array}$ & & $0.0015^{* *}$ & & $0.2561^{\mathrm{ns}}$ & & $0.0029 * *$ & ns & $0.0003 * * *$ \\
\hline
\end{tabular}

$*, * *, * * *, * * * * \mathrm{~F}$ values significant at the $\mathrm{P}=0.05, \mathrm{P}=0.01, \mathrm{P}=0.001$ and $\mathrm{P} \leq 0.0001$ levels, respectively. $\mathrm{ns}$ means non-significant at the $\mathrm{P}=0.05$ level.

${ }^{a}$ Data are average of three replicates. Different letters indicate statistical significance at the $\mathrm{P}=0.05$ level within the same column and same conditions of cultivation.

Fig.1 Percent colonization in rice varieties at 30, 45 and 60 DAS

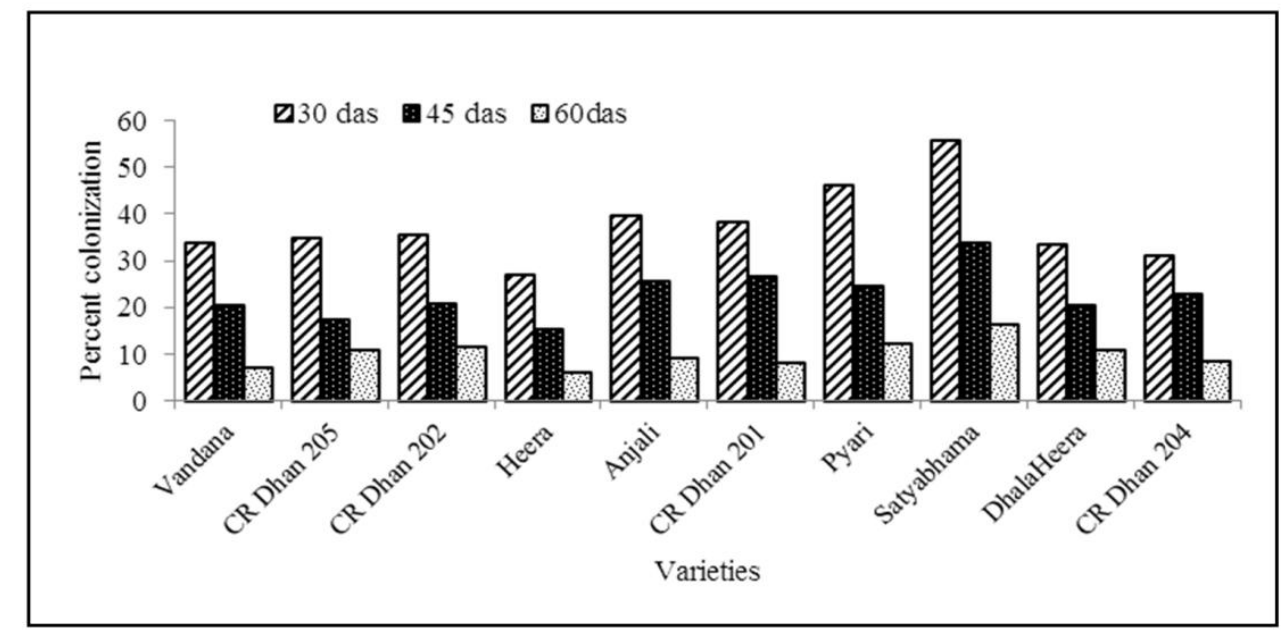


Fig.2 Correlation analyses of percent colonization with A. Total N uptake, B. Total P uptake, C. Total K uptake, D. Total Zn uptake and E. Total Fe uptake of rice plants
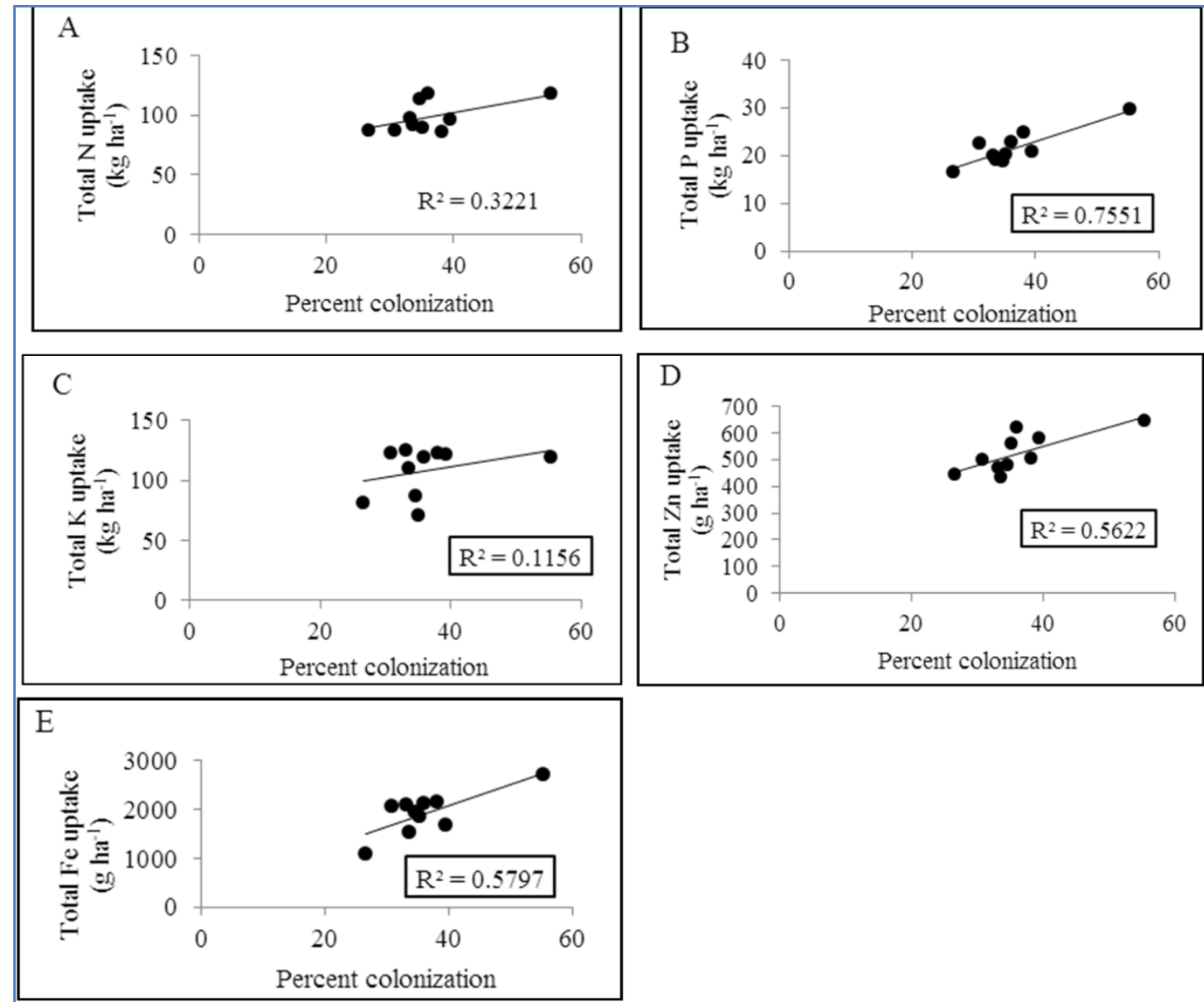

Fig.3 Shoot dry weight as recorded on 30DAS and at the time of harvest

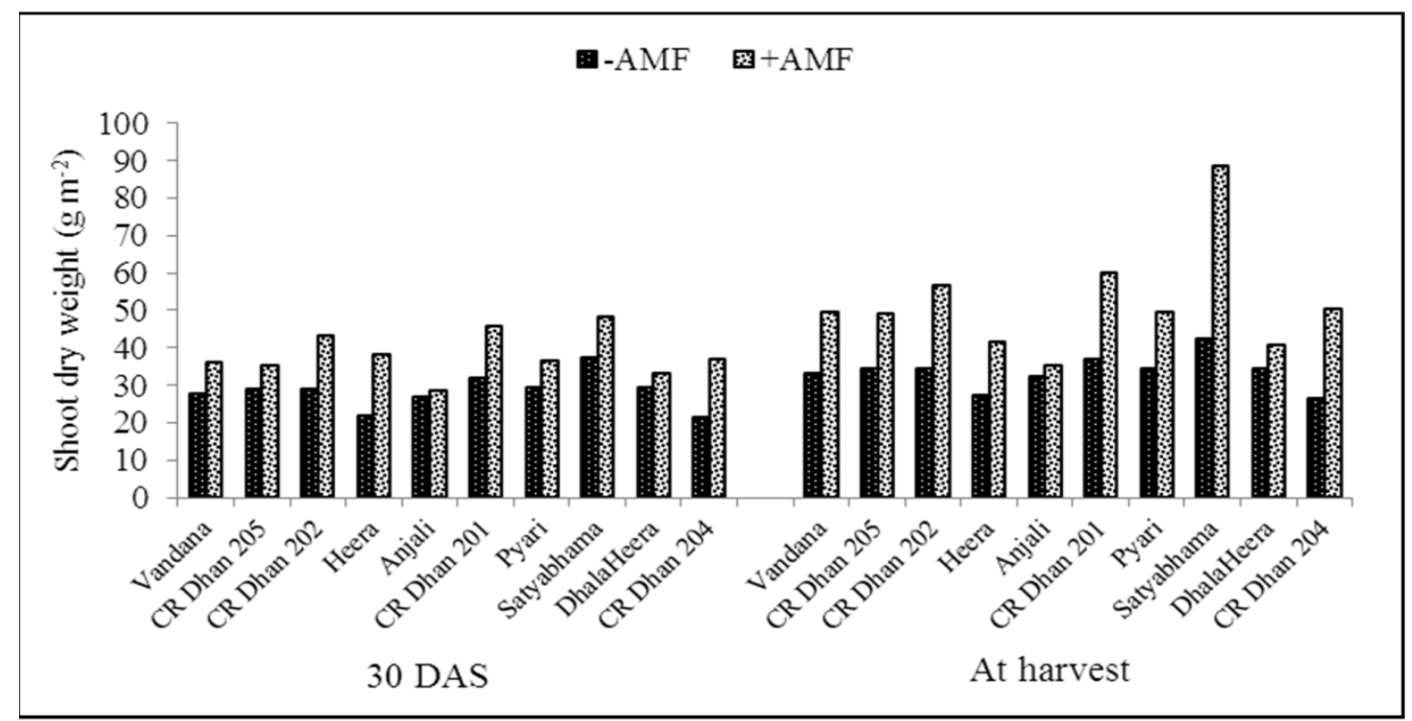


Fig.4 Protein content in grains at the time of harvest of rice varieties

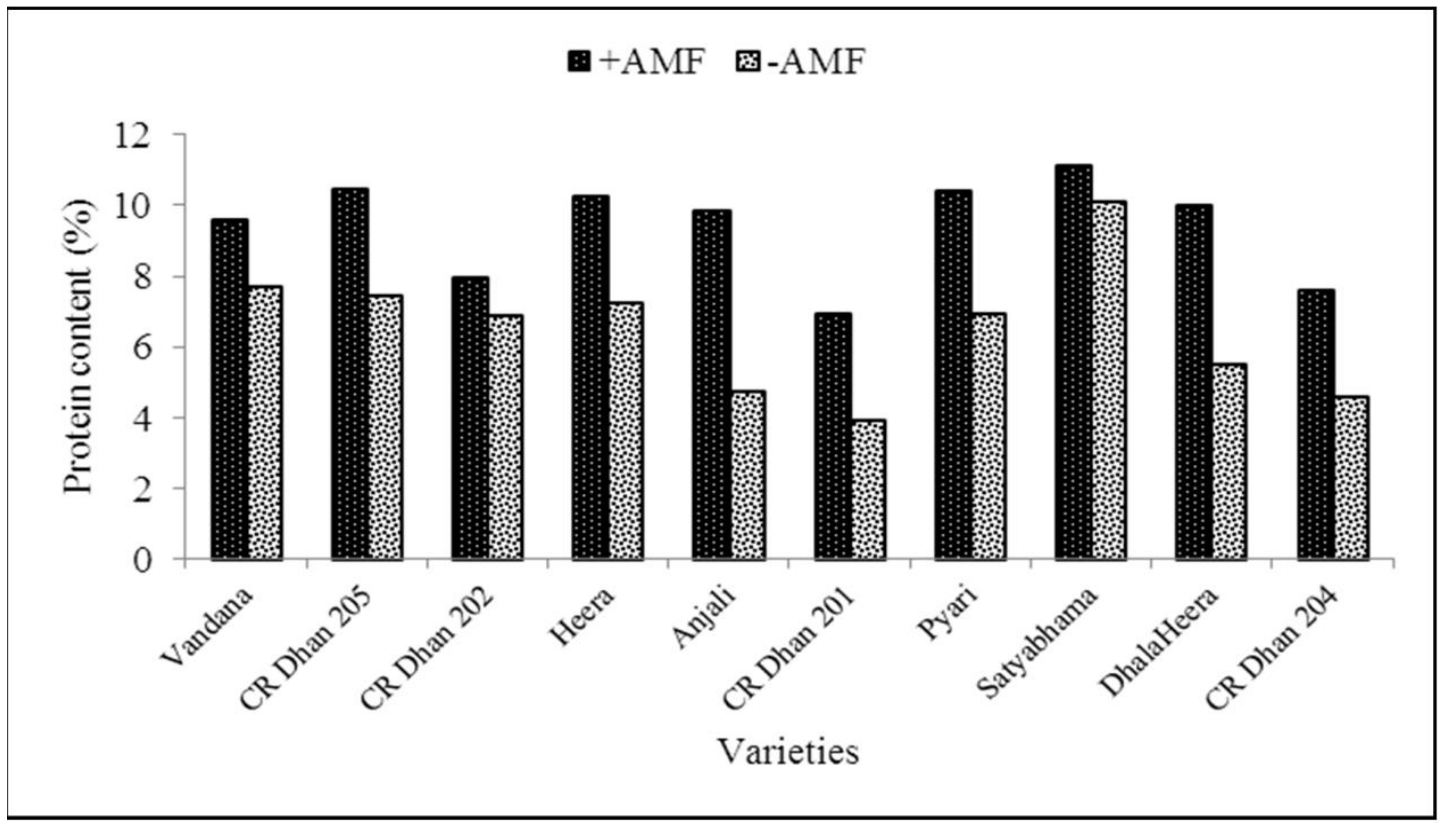

Fig.5 Correlation analyses of percent colonization with A. Shoot dry weight, B. Total chlorophyll content, and C. NR activity of rice plants
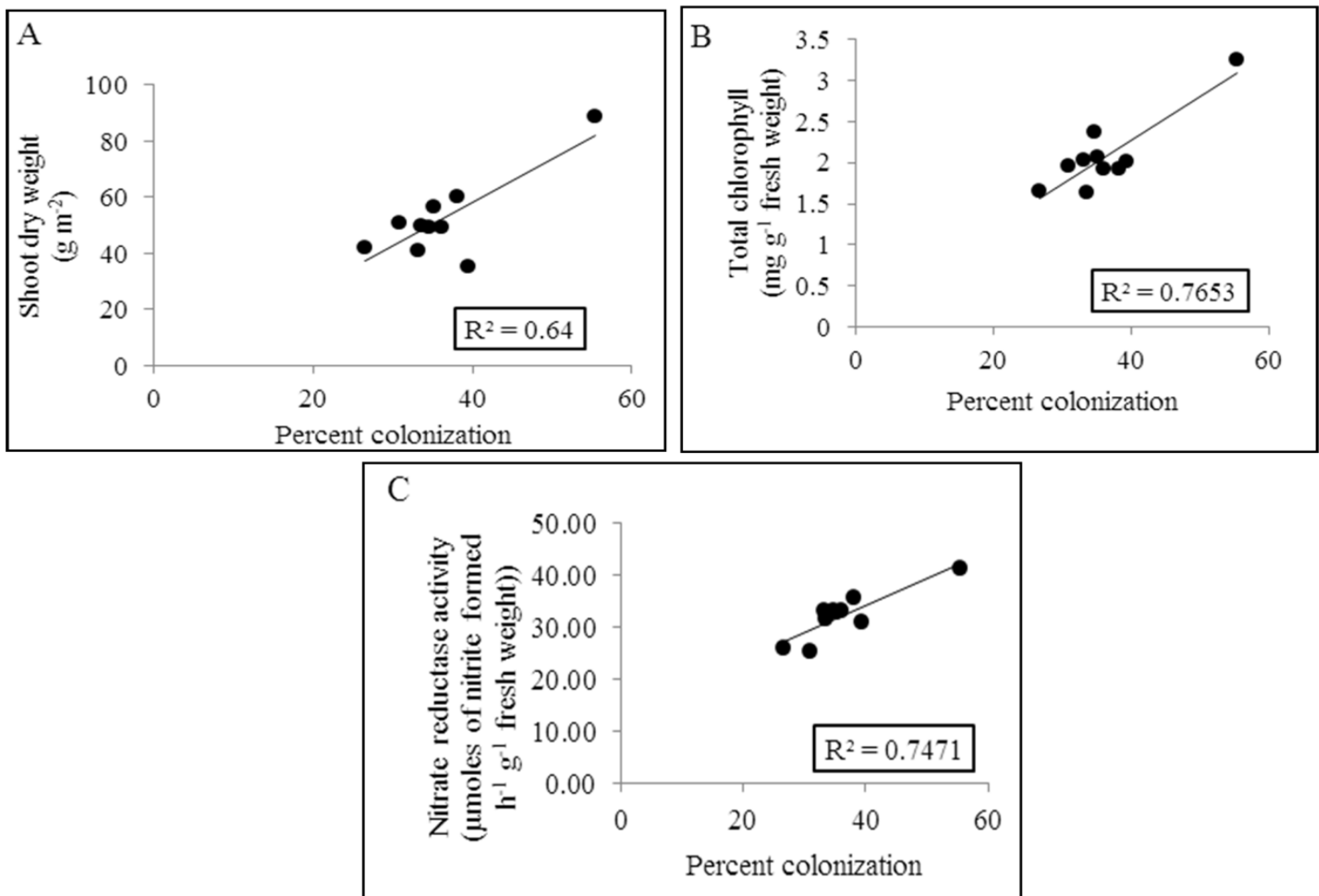
Yields obtained with ARS varieties vary from 3.5 to $6.5 \mathrm{t} / \mathrm{ha}$, which is about $20-30 \%$ lesser than that obtained with lowland varieties grown under flooded conditions (Farooq et al., 2009). Mycorrhizal symbioses are mutualistic interactions between the root systems of around $80 \%$ of land plants and the mycelium of various fungi (Wang and Qiu, 2006). Mycorrhizal fungi participate actively to plant development (Smith and Read, 2008) by improvement of access to nutrients, particularly when resources become scarce. In our study, we demonstrated the potential effect of AMF colonization of rice roots in the earlier growth stage on the nutrient uptake in grain and straw of rice varieties grown under aerobic conditions. We also demonstrated the positive effect of AMF treatment on the total chlorophyll content and NR activity at flag leaf stage of rice varieties.

Maximum root colonization was observed at 30 DAS. According to Miller (2000), during the early phase of the crop cycle, when roots are not yet well developed, AM symbiosis can make an important contribution to plant growth by enhancing nutrient acquisition. Two rice varieties, Satyabhama and Pyari, were highly responsive towards AMF treatment showing maximum root colonization by AMF over other rice varieties at 30, 45 and 60 DAS. All the rice varieties differed significantly with respect to nitrogen $(\mathrm{N})$, phosphorus $(\mathrm{P})$, potassium $(\mathrm{K})$, zinc $(\mathrm{Zn})$ and iron $(\mathrm{Fe})$ concentration in the grain and straw and also their uptake in grain or straw or their total.

Nitrogen concentration in grain and straw and its uptake in grain, straw and their total was found to be significantly higher in AMF treated rice. The positive involvement of AMF in enhanced nitrogen uptake and assimilation by host plant is well documented (Cavagnaro et al., 2012; Lee et al., 2012). Hawkins et al., (2000) reported that AM fungi are able to absorb glycine and glutamic acid and transport nitrogen from these sources to the host plants.

Phosphorus concentration in grain and straw was found to be significantly higher in AMF treated rice plants compared to the control plants. As a result of AMF treatment, $\mathrm{P}$ uptake was significantly increased in grain, straw and their total. $P$ plays a very important role in photosynthesis; $\mathrm{P}$ is crucial for activation of ribulose-1,5-bisphosphate carboxylase oxygenase (Rubisco) and in providing phosphorylated intermediates of Calvin cycle (Hernández and Munné-Bosch, 2015).

Similarly, K accumulation was recorded higher in AMF treated rice. A higher level of $\mathrm{K}$ accumulation in plants inoculated with AM fungi is well reported in different crops (Borah and Phukan, 2006; Rajesh et al., 2011: Baslam et al., 2013). Maintaining an elevated level of $\mathrm{K}$ in plant cells is important for running physiological process like plasma membrane polarization, growth, stomatal aperture or adaptation to adaptation to environmental changes (Anschütz et al., 2014; Shabala and Pottosin, 2014; Benito et al., 2014) and for improvement in hydric stress tolerance (Wu and Xia 2006; ElMesbahi et al., 2012). These results indicate that AM fungi enhance growth and stress tolerance in rice under aerobic conditions which is accompanied by intermittent drying periods that can be harmful for the growth of rice plants. A significant correlation between $\mathrm{K}$ and $\mathrm{P}$ during $\mathrm{AM}$ symbiosis has been reported by Olsson et al., (2011); results obtained in our study upholds this correlation since rice plants inoculated with AMF displayed significantly higher $\mathrm{P}$ and $\mathrm{K}$ content as compared to the uninoculated rice plants. Gao et al., (2007) studied the effect of AMF inoculation on growth performance and $\mathrm{Zn}$ uptake by rice genotypes varying in $\mathrm{Zn}$ uptake 
when non mycorrhizal and showed that AMF inoculated plants produced more biomass and took up more $\mathrm{Zn}$ than non-mycorrhizal controls.

Saia et al., (2014) reported higher biomass production under water stressed conditions in Berseem, clover on AM symbiosis. Increased biomass may be attributed to the enhancement of nutrient uptake offered by AM fungi during active growth stage under aerobic conditions, which satisfied both rice and fungal nutritional needs for growth.

The interaction between the AMF treatment and rice varieties shows that AMF significantly contributed towards nutrient concentration and uptake in rice plants under aerobic conditions. This is also evident by the observation that Satyabhama and Pyari which were recorded for higher root colonization were also reported to have maximum nutrient concentration in grain and straw and enhanced nutriet uptake by rice plants.

In aerobic system of rice cultivation, ammonia is nitrified in the oxidized environment and hence, nitrate is the dominant form of available $\mathrm{N}$ (Bouman et al., 2002). An analysis of nitrate reductase (NR) activity in flag leaves was undertaken because this enzyme is involved in nitrogen assimilation by plants. AMF treated rice plants showed higher values of NR activity than the control plants. The AM-upland rice varieties showed about $103 \%$ enhanced NR activity than control rice plants. Interestingly, the upland rice genotypes (Pyari and Satyabhama) with highest NR also recorded the highest shoot dry weight and $\mathrm{N}$ content compared to uninoculated plants. The interaction between rice genotypes and AM fungi is highly significant $(P=0.0001)$ for NR activity which indicates that mycorrhizal inoculation strongly affected the improvement of NR activity in AM plants.
In the present study, the total chlorophyll content was higher in AMF treated rice plants; this illustrates better photosynthetic efficiency which can be attributed to the better growth observed in AM- colonized plants. A positive correlation between percent colonization and total chlorophyll content $\left(R^{2}=0.76\right)$. It has been shown that under water stress conditions, the AM-colonized plants show enhanced photosynthetic efficiency and thus increase in rice shoot biomass (RuizSanchez et al., 2010). The higher values of chlorophyll content in AMF treated upland rice plants indicate that the photosynthetic apparatus was less affected by the hydric stress generated during intermittent drying periods (Germ et al., 2005). Higher amount of chlorophyll also stimulates production of increased amount of photosynthates in the host plant, which would provide more substrate for the growth and development of root system, AM fungal consortia and rhizospheric microflora (Wu et al., 2010; Li et al., 2017; Hassan et al., 2017). AMF significantly increase the net photosynthesis by increasing the total chlorophyll (Manoharan et al., 2008; Thamizhiniyan et al., 2009). Arbuscularmycorrhizal fungi (AMF) act as a carbon sink as they rely on plant photo assimilates, and may stimulate the photosynthesis rate of the host plant depending on the environmental conditions (Kaschuk et al., 2009). Although there is evidence that the degree of AM root colonization is not necessarily linked to the plant growth responses (Marulanda et al., 2003), in this study we found a positive correlation between the mycorrhizal root lengths achieved and plant growth stimulation. The results obtained in the present study regarding plant nutrient uptake and concentration in grain and straw and protein content in grain evidently indicates that enhanced chlorophyll content and NR activity of rice treated with AMF especially during the peak vegetative growth phase has 
led to the better plant health and yield response, under aerobic conditions.

This study demonstrated the beneficial effects AM association in rice plants under aerobic conditions. There is considerable potential for using AMF to increase production of aerobic rice. Moreover, AM symbiosis made an important contribution during the early growth phase, when nutrient uptake is limited by the relatively less developed plant root system. We showed that AM association in the early growth stages of rice can have important impact on the nutrient concentration and uptake in later growth stages. Although, mycorrhizal symbiosis could not completely compensate for large yield gap of aerobic rice when compared with flooded rice; but can be considered an important plant strategy for maintaining and improving rice growth under aerobic conditions

\section{References}

Adak A., Prasanna R., Babu S., Bidyarani N., Verma S., Pal M., Shivay Y. S., Nain L. 2016. Micronutrient enrichment mediated by plant-microbe interactions and rice cultivation practices. Journal of Plant Nutrition 39:1216-1232.

Anschütz U., Becker D., Shabala S. 2014. Going beyond nutrition: regulation of potassium homoeostasis as a common denominator of plant adaptive responses to environment. Journal of Plant Physiology 171:670-687.

Azcón R., Barea J.-M. 2010. Mycorrhizosphere interactions for legume improvement. Pages 237-271. Microbes for Legume Improvement, Springer.

Barea J. M., Azcón R., Azcón-Aguilar C. 2005. Interactions between mycorrhizal fungi and bacteria to improve plant nutrient cycling and soil structure.
Pages 195-212. Microorganisms in soils: roles in genesis and functions, Springer.

Baslam M., Garmendia I., Goicoechea N. 2013. The arbuscular mycorrhizal symbiosis can overcome reductions in yield and nutritional quality in greenhouse-lettuces cultivated at inappropriate growing seasons. Scientia Horticulturae 164:145-154.

Benito B., Haro R., Amtmann A., Cuin T. A., Dreyer I. 2014. The twins $\mathrm{K}+$ and $\mathrm{Na}+$ in plants. Journal of Plant Physiology 171:723-731.

Bouman B. 2001. Water-efficient management strategies in rice production. Int. Rice. Res. Notes 16:1722.

Bouman B., Wang H., Yang X., Zhao J., Wang C. 2002. Aerobic rice (Han Dao): a new way of growing rice in watershort areas. Pages 31. Proceedings of the 12th international soil conservation organization conference: Tsinghua University Press Beijing, China.

Castaneda A., Bouman B., Peng S., Visperas R. 2002. The potential of aerobic rice to reduce water use in water-scarce irrigated lowlands in the tropics. Waterwise rice production. Pp. 8-11.

Cavagnaro T., Barrios-Masias F., Jackson L. 2012. Arbuscular mycorrhizas and their role in plant growth, nitrogen interception and soil gas efflux in an organic production system. Plant and Soil 353:181-194.

El-Mesbahi M. N., Azcón R., Ruiz-Lozano J. M., Aroca R. 2012. Plant potassium content modifies the effects of arbuscular mycorrhizal symbiosis on root hydraulic properties in maize plants. Mycorrhiza 22:555-564.

Farooq M., Kobayashi N., Wahid A., Ito O., Basra S. M. 2009. Retracted: Chapter 6 strategies for producing more rice with less water: Elsevier. 
Gao X., Kuyper T. W., Zou C., Zhang F., Hoffland E. 2007. Mycorrhizal responsiveness of aerobic rice genotypes is negatively correlated with their zinc uptake when nonmycorrhizal. Plant and Soil 290:283-291.

Germ M., Kreft I., Osvald J. 2005. Influence of UV-B exclusion and selenium treatment on photochemical efficiency of photosystem II, yield and respiratory potential in pumpkins (Cucurbita pepo L.). Plant Physiology and Biochemistry 43:445-448.

Hageman R., Hucklesby D. 1971. [45] Nitrate reductase from higher plants. Pages 491-503. Methods in Enzymology, vol. 23 Elsevier.

Hawkins H.-J., Johansen A., George E. 2000. Uptake and transport of organic and inorganic nitrogen by arbuscular mycorrhizal fungi. Plant and Soil 226:275-285.

Hernández I., Munné-Bosch S. 2015. Linking phosphorus availability with photooxidative stress in plants. Journal of Experimental Botany 66:2889-2900.

Hiscox J. T., Israelstam G. 1979. A method for the extraction of chlorophyll from leaf tissue without maceration. Canadian Journal of Botany 57:13321334.

Ilag L. L., Rosales A., Elazegui F., Mew T. 1987. Changes in the population of infective endomycorrhizal fungi in a rice-based cropping system. Plant and Soil 103:67-73.

Jackson M. L. 1958. Soil chemical analysis. Prentice-Hall, Inc.; Englewood Cliffs.

Jez J. M., Lee S. G., Sherp A. M. 2016. The next green movement: plant biology for the environment and sustainability. Science 353:1241-1244.

Kaschuk G., Kuyper T. W., Leffelaar P. A., Hungria M., Giller K. E. 2009. Are the rates of photosynthesis stimulated by the carbon sink strength of rhizobial and arbuscular mycorrhizal symbioses? Soil Biology and Biochemistry 41:12331244.

Kijne J. W., Barker R., Molden D. J. 2003. Water productivity in agriculture: limits and opportunities for improvement. Cabi.

Kumar V., Ladha J. K. 2011. Direct seeding of rice: recent developments and future research needs. Pages 297-413. Advances in Agronomy, vol. 111 Elsevier.

Lee B.-R., Muneer S., Avice J.-C., Jung W.J., Kim T.-H. 2012. Mycorrhizal colonisation and P-supplement effects on $\mathrm{N}$ uptake and $\mathrm{N}$ assimilation in perennial ryegrass under well-watered and drought-stressed conditions. Mycorrhiza 22:525-534.

Li, H. L., Qiang, S. W. 2017. Mycorrhizas promote plant growth, root morphology and chlorophyll production in white clover. Biotechnology, 16: 34-39.

Lin S., Dittert K., Tao H., Kreye C., Xu Y., Shen Q., Fan X., Sattelmacher B. 2002. The ground-cover rice production system (GCRPS): a successful new approach to save water and increase nitrogen fertilizer efficiency. WaterWise Rice Production. IRRI, LosBaños, Philippines:187-196.

Manoharan P., Pandi M., Shanmugaiah V., Gomathinayagam S., Balasubramanian N. 2008. Effect of vesicular arbuscular mycorrhizal fungus on the physiological and biochemical changes of five different tree seedlings grown under nursery conditions. African Journal of Biotechnology 7.

Marulanda A., Azcon R., Ruiz- Lozano J. M. 2003. Contribution of six arbuscular mycorrhizal fungal isolates to water uptake by Lactuca sativa plants under drought stress. Physiologia Plantarum 119:526-533. 
Miller S. P. 2000. Arbuscular mycorrhizal colonization of semi-aquatic grasses along a wide hydrologic gradient. The New Phytologist 145:145-155.

Nishio M., Kusano S. 1975. Effect of root residues on the growth of upland rice. Soil Science and Plant Nutrition 21:391-395.

Nishizawa T. 1971. Survey of the nematode population in the experimental fields of successive or rotative plantation. Pages 121-122. Proc. Kanto-Tosan Plant Prot. Soc.

Olsson P. A., Hammer E. C., Pallon J., Van Aarle I. M., Wallander H. 2011. Elemental composition in vesicles of an arbuscular mycorrhizal fungus, as revealed by PIXE analysis. Fungal Biology 115:643-648.

Peng S., Bouman B., Visperas R. M., Castañeda A., Nie L., Park H.-K. 2006. Comparison between aerobic and flooded rice in the tropics: agronomic performance in an eight-season experiment. Field Crops Research 96:252-259.

Phillips J. M., Hayman D. 1970. Improved procedures for clearing roots and staining parasitic and vesiculararbuscular mycorrhizal fungi for rapid assessment of infection. Transactions of the British Mycological Society 55:158IN118.

Prasad R. 2006. Zinc in soils and in plant, human \& animal nutrition. Indian Journal of Fertilisers 2:103.

Prasad R. 2011. Aerobic rice systems. Pages 207-247. Advances in Agronomy, vol. 111 Elsevier.

Prasanna R., Adak A., Verma S., Bidyarani N., Babu S., Pal M., Shivay Y. S., Nain L. 2015. Cyanobacterial inoculation in rice grown under flooded and SRI modes of cultivation elicits differential effects on plant growth and nutrient dynamics. Ecological Engineering 84:532-541.

Rajesh K., Sandeep C., Suresh C. 2011. Microbial, biochemical, anatomical and histochemical analysis of black pepper and sorghum inoculated with mycorrhiza. Journal of Phytology, 3.

Ruiz-Sánchez M., Aroca R., Muñoz Y., Polón R., Ruiz-Lozano J. M. 2010. The arbuscular mycorrhizal symbiosis enhances the photosynthetic efficiency and the antioxidative response of rice plants subjected to drought stress. Journal of Plant Physiology 167:862869.

Saia S., Amato G., Frenda A. S., Giambalvo D., Ruisi P. 2014. Influence of arbuscular mycorrhizae on biomass production and nitrogen fixation of berseem clover plants subjected to water stress. PloS one 9:e90738.

SAS 9.3, SAS Institute, Inc. Cary, NC, USA, 1990.

Secilia J., Bagyaraj D. 1994. Selection of efficient vesicular-arbuscular mycorrhizal fungi for wetland rice - a preliminary screen. Mycorrhiza 4:265268.

Shabala S., Pottosin I. 2014. Regulation of potassium transport in plants under hostile conditions: implications for abiotic and biotic stress tolerance. Physiologia Plantarum 151:257-279.

Singh Y. 2013. Crop and water productivity as influenced by rice cultivation methods under organic and inorganic sources of nutrient supply. Paddy and Water Environment 11:531-542.

Smith S. E., Read D. J. 2008. Mycorrhizal symbiosis. 3rd. Academic Press New York.

Solaiman M. Z., Hirata H. 1996. Effectiveness of arbuscular mycorrhizal colonization at nursery-stage on growth and nutrition in wetland rice (Oryza sativa L.) after transplanting under 
different soil fertility and water regimes.

Soil Science and Plant Nutrition 42:561-571.

Thamizhiniyan P., Panneerselvam M., Lenin M. 2009. Studies on the growth and biochemical activity of Coleus aromaticus Benth. as influenced by AM fungi and Azospirillum. Recent Research in Science and Technology 1.

Trouvelot A., Kough J., Gianinazzi-Pearson V. 1986. Mesure du taux de mycorhization VA d'un système radiculaire. Recherche de méthodes d'estimation ayant une signification fonctionnelle. Physiological and genetical aspects of mycorrhizae $=$ Aspects physiologiques et genetiques des mycorhizes: proceedings of the 1st European Symposium on Mycorrhizae, Dijon, 1-5 July 1985: Paris: Institut national de le recherche agronomique, c1986.

Tuong T., Bouman B. 2003. Rice production in water-scarce environments. Water productivity in agriculture: Limits and opportunities for improvement 1:13-42.

Vallino M., Greppi D., Novero M., Bonfante P., Lupotto E. 2009. Rice root colonisation by mycorrhizal and endophytic fungi in aerobic soil. Annals of Applied Biology 154:195-204.

Ventura W., Watanabe I., Castillo M. B., De la Cruz A. 1981. Involvement of nematodes in the soil sickness of a dryland rice-based cropping system. Soil Science and Plant Nutrition 27:305-315.

Wang B., Qiu Y. L. 2006. Phylogenetic distribution and evolution of mycorrhizas in land plants. Mycorrhiza 16:299-363.

Wu Q. S., Xia R. X. 2006. Arbuscular mycorrhizal fungi influence growth, osmotic adjustment and photosynthesis of citrus under well-watered and water stress conditions. Journal of Plant Physiology 163:417-425.

Wu Q. S., Zou Y. N., He X. H. 2010. Contributions of arbuscular mycorrhizal fungi to growth, photosynthesis, root morphology and ionic balance of citrus seedlings under salt stress. Acta Physiologiae Plantarum 32:297-304.

Zare M. H., Khanpour A. N., Ghanati F. 2017. Influence of mycorrhizal fungi on growth, chlorophyll content, and potassium and magnesium uptake in maize. Journal of Plant Nutrition 40:2026-2032.

\section{How to cite this article:}

Ekta Narwal, K. Annapurna, Jairam Choudhary and Seema Sangwan. 2018. Effect of Arbuscular mycorrhizal Fungal Colonization on Nutrient Uptake in Rice Aerobic Conditions. Int.J.Curr.Microbiol.App.Sci. 7(04): 1072-1093. doi: https://doi.org/10.20546/ijcmas.2018.704.118 